ANNALES

POLONICI MATHEMATICI

$94.2(2008)$

\title{
On the Green function on a certain class of hyperconvex domains
}

\author{
by Gregor Herbort (Wuppertal)
}

\begin{abstract}
We study the behavior of the pluricomplex Green function on a bounded hyperconvex domain $D$ that admits a smooth plurisubharmonic exhaustion function $\psi$ such that $1 /|\psi|$ is integrable near the boundary of $D$, and moreover satisfies the estimate $|\psi| \leq C \exp \left(-C^{\prime}\left(\log \left(1 / \delta_{D}\right)\right)^{\alpha}\right)$ at points close enough to the boundary with constants $C, C^{\prime}>0$ and $0<\alpha<1$. Furthermore, we obtain a Hopf lemma for such a function $\psi$. Finally, we prove a lower bound on the Bergman distance on $D$.
\end{abstract}

1. Introduction. In $1985 \mathrm{M}$. Klimek introduced the pluricomplex Green function of a bounded domain $D \subset \mathbb{C}^{n}$. It is defined by

$$
\begin{aligned}
\mathscr{G}_{D}(z, w)=\sup \{u(z) \mid u: D \rightarrow[-\infty, 0), u \in \operatorname{PSH}(D), \\
u(z)-\log |z-w| \text { is bounded from above near } w\},
\end{aligned}
$$

where $\operatorname{PSH}(D)$ denotes the family of plurisubharmonic functions on $D$. In [18] and [9] the important properties of the Green function were established and also its relationship to the complex Monge-Ampère equation was clarified.

The Green function is a powerful tool for investigations in Bergman theory, when one wants to construct good holomorphic square-integrable functions by means of the $\bar{\partial}$-technique with weights (see for example $[5,12,15$, 17]). On a hyperconvex domain it is known from $[9,18]$ that $\mathscr{G}_{D}(z, w) \rightarrow 0$ as $z$ tends to the boundary and $w$ is kept fixed. A domain $D \subset \mathbb{C}^{n}$ is called hyperconvex ([21]) if it admits a bounded continuous plurisubharmonic exhaustion function $\psi: D \rightarrow(-1,0)$.

When using the Green function as a weight, one needs, however, information on the sublevel sets of the Green function $\mathscr{G}_{D}(\cdot, w)$ as $w$ tends to the boundary; more precisely, it is desirable to describe, in terms of the boundary distance of the pole $w$, where the sets $\left\{\mathscr{G}_{D}(\cdot, w)<-1\right\}$ are situated.

2000 Mathematics Subject Classification: 32U35, 32F45.

Key words and phrases: pluricomplex Green function, hyperconvex domain, Demailly regularization, Bergman distance. 
This is a difficult question, since for $n>1$ the pluricomplex Green function is no longer symmetric (see [2]). Recently, some progress has been made in this direction: see for example $[5,12,16]$.

Carlehed, Cegrell and Wikström [8] obtained a first result on the behavior of the Green function $\mathscr{G}_{D}(\cdot, w)$ as $w$ approaches a boundary point $w_{0}$ : If $\left(w_{j}\right)_{j}$ is a sequence of points in $D$ that tends towards $w_{0}$, then there exists a pluripolar set $E$ such that

$$
\limsup _{j \rightarrow \infty} \mathscr{G}_{D}\left(z, w_{j}\right)=0 \quad \text { for } z \in D \backslash E .
$$

We prove that under a mild additional condition on $D$ (which is considerably weaker than those from [12] and [16]), the set $E$ is empty and the $\lim \sup$ is in fact a limit. We assume in Sections 2 through 6 that $n>1$. The case $n=1$ will be discussed in Section 7 .

Theorem 1.1. Let $D$ be a bounded hyperconvex domain in $\mathbb{C}^{n}$ with $n \geq 2$ that admits a plurisubharmonic smooth exhaustion function $\psi: D \rightarrow[-1,0)$ with the following two properties:

(1) There is a positive measurable function $h$ such that $h^{-1 /(n-1)}$ is integrable over $D$ (with respect to the Lebesgue measure) and

$$
\left(d d^{c} \psi\right)^{n} \geq h\left(d d^{c}|z|^{2}\right)^{n} .
$$

(2) There are constants $1>\alpha>0$ and $\widehat{C}_{1}, \widehat{C}_{2}>0$ such that

$$
\psi \geq-\widehat{C}_{1} \exp \left(-\widehat{C}_{2}\left(\log \frac{1}{\delta_{D}}\right)^{\alpha}\right)
$$

on $D \cap\left\{\delta_{D}<1\right\}$, where $\delta_{D}$ denotes the boundary distance function on $D$.

Then there are constants $\widetilde{C}, \delta_{0}>0$ such that for any compact subset $K \subset D$ and $w \in D \backslash K$ with $\delta_{D}(w) \leq \min \left\{\delta_{0}, \delta_{D}(K) / 4\right\}$,

$$
\sup _{z \in K}\left|\mathscr{G}_{D}(z, w)\right| \leq \widetilde{C}\left(\frac{|\psi(w)|^{1 / 3 n}}{\delta_{D}(K)^{2 n+3}}+\delta_{D}(w)\right) .
$$

A function with property (1) of the above theorem exists on a general hyperconvex domain, as follows from a result of [6]:

Theorem 1.2. Let $D$ be a bounded hyperconvex domain. Given a continuous function $f$ on $\partial D$ that extends to a plurisubharmonic function on $D$, and a continuous function $F: \bar{D} \rightarrow[0, \infty)$, there exists a uniquely determined continuous function $u=u_{(f, F)}$ on $\bar{D}$ that is plurisubharmonic on $D$ and such that $u=f$ on $\partial D$, and $\left(d d^{c} u\right)^{n}=F d z_{1} \wedge d \bar{z}_{1} \wedge \cdots \wedge d z_{n} \wedge d \bar{z}_{n}$ on $D$.

The uniquely determined solution $\varphi_{D}$ from Theorem 1.2 which corresponds to the data $f=0, F=1$ will of course satisfy the requirement (1). 
With slight changes the method used to prove Theorem 1.1 yields

Theorem 1.3. Let $n \geq 2$. Suppose that $D \subset \subset \mathbb{C}^{n}$ is hyperconvex and admits a smooth plurisubharmonic exhaustion function $\psi: D \rightarrow(-1,0)$ that satisfies with some constant $C_{1}>0$ the estimate

(3) $\frac{1}{C_{1}}\left(\log \frac{1}{\delta_{D}}\right)^{-M} \leq|\psi| \leq C_{1}\left(\log \frac{1}{\delta_{D}}\right)^{-N}$

on $D \cap\left\{\delta_{D}<1\right\}$ with exponents $M, N>2(n+1)$ such that $N \leq M \leq$ $-1+N^{2} / 4 n$. Then there exist constants $\widetilde{C}, \delta_{*}>0$ such that for any compact set $K \subset D$ and any $w \in D \backslash K$ with $\delta_{D}(w) \leq \min \left\{\delta_{*}, \delta_{D}(K) / 4\right\}$,

$$
\sup _{z \in K}\left|\mathscr{G}_{D}(z, w)\right| \leq \widetilde{C}\left(\frac{|\psi(w)|^{\gamma(n-1) / n}}{\delta_{D}(K)^{2 n}}+|\psi(w)|^{\beta_{1}(n-1) / n}\right),
$$

where $\beta=\frac{1}{2}\left(N(n-1) / n^{2}-M^{\prime}+1 / N^{\prime}\right), N^{\prime}=N(1-1 / n), M^{\prime}=M(1-1 / n)$, $\gamma=n \beta / 2 N(n-1)$, and $\beta_{1}=\min \{\beta, 1 / n-\gamma\}$. Note that $\beta \geq N / 48 n$ for $n \geq 2$.

Under the hypotheses of Theorem 1.1 a lower bound on the Bergman distance can be obtained (using the idea of [14]):

TheOREM 1.4. Let $D$ and $\psi$ be as in Theorem 1.1. Then there exists a constant $C_{*}>0$ such that for any fixed $P \in D$,

$$
d_{D}^{\mathrm{B}}(Q, P) \geq C_{*} \log \log \log \frac{1}{|\psi(Q)|}
$$

whenever $\delta_{D}(Q) \ll 1$.

Note that no Hölder condition on the exhaustion function $\psi$ is needed.

Acknowledgements. I would like to express my thanks to the referee for a patient and careful reading of my manuscript. His suggestions were a valuable help for me to remove defects in the statements and proofs in this article.

\section{Lower bounds on the Demailly regularization of a plurisub- harmonic function}

Some notations. Let $D \subset \subset \mathbb{C}^{n}$ be a pseudoconvex domain and $V$ a negative plurisubharmonic function on $D$. Following the method of Demailly [10] we regularize $V$. For this let $m>0$ be a positive number and $\mathcal{H}_{2 m V}(D)$ the Hilbert space of holomorphic functions in $D$ such that the weighted $L^{2}$-norm

$$
\|f\|_{2 m V}:=\left(\int_{D}|f|^{2} e^{-2 m V} d^{2 n} z\right)^{1 / 2}
$$


is finite. We denote by $K_{D, 2 m V}$ the Bergman kernel associated to $\mathcal{H}_{2 m V}$. Then the function

$$
V_{m}(z):=\frac{1}{2 m} \log K_{D, 2 m V}(z, z)
$$

is plurisubharmonic, and we have

$$
V_{m}(z):=\frac{1}{2 m} \sup \left\{\log |f(z)|^{2} \mid f \in \mathcal{H}_{2 m V},\|f\|_{2 m V} \leq 1\right\} .
$$

It is shown in Proposition 3.1 of [10] that, with suitable constants $C_{1}, C_{2}$, for any $z \in D$ and any $0<r<\delta_{D}(z)$ one has

$$
V(z)-\frac{1}{C_{1} m} \leq V_{m}(z) \leq \sup _{x \in B(z, r)} V(x)+\frac{1}{m} \log \frac{C_{2}}{r^{n}},
$$

and moreover $V_{m}$ tends to $V$ pointwise and in the $L_{\text {loc-topology on } D \text { as }}^{1}$ $m \rightarrow \infty$.

For $z^{0} \in D$ and $r \in\left(0, \delta_{D}\left(z^{0}\right)\right)$, and a measurable function $V$ on $D$, we denote by $M\left[V, z^{0}, r\right]$ the spherical mean of $V$ over $\partial B\left(z^{0}, r\right)$ and by $A\left[V, z^{0}, r\right]$ the average of $V$ over the full ball $B\left(z^{0}, r\right)$.

For any subharmonic function $V$ on $D$ one has

$$
A\left[V, z^{0}, r\right] \leq M\left[V, z^{0}, r\right] .
$$

Given $z^{0} \in D$, we need a lower estimate for $V_{m}\left(z^{0}\right)$ in terms of the mass of the ball $B\left(z^{0}, r\right)$ with respect to the measure $\Delta V$. Then we can apply the result to the case where $V$ is the Green function with a pole at $w \in D$. This together with an estimate of Błocki will help control the value $V_{m}\left(z^{0}\right)$ as $w$ tends to a boundary point.

We begin with a comparison lemma for the weighted Bergman kernel.

Lemma 2.1. Let $0<r<\delta_{D}\left(z^{0}\right) / 2$. Then:

(a) We have

$$
K_{2 m V}\left(z^{0}, z^{0}\right) \geq C_{D} \frac{1}{A\left[e^{-2 m V}, z^{0}, r\right]},
$$

where $C_{D}>0$ depends only on the diameter of $D$.

(b) The regularization $V_{m}$ can be estimated from below by

$$
V_{m}\left(z^{0}\right) \geq-\frac{\log C_{D}}{2 m}-\frac{1}{2 m} \log \left(A\left[e^{-2 m V}, z^{0}, r\right]\right) .
$$

Proof. We choose a cut-off function $\chi \in C^{\infty}(\mathbb{R})$ with $\chi(s)=1$ for $s \leq 1 / 4$ and $\chi(s)=0$ for $s>9 / 16$. Then the $(0,1)$-form

$$
v=\bar{\partial}\left(\chi\left(\frac{\left|z-z^{0}\right|^{2}}{r^{2}}\right)\right)=\chi^{\prime}\left(\frac{\left|z-z^{0}\right|^{2}}{r^{2}}\right) \frac{\bar{\partial}\left|z-z^{0}\right|^{2}}{r^{2}}
$$


is smooth and $\bar{\partial}$-closed on $D$. We will solve a suitable $\bar{\partial}$-problem for these data on $D$ with weight function

$$
\varphi(z):=2 m V(z)+2 n \log \left|z-z^{0}\right|
$$

Our plan is to use the $L^{2}$-technique developed in [20,3] (see also [13]). The relevant tool for solving $\bar{\partial}$ will be the following slight modification of Lemma 2.2 from [17]:

Lemma 2.2. Let $\Omega \subset \subset \mathbb{C}^{n}$ be a pseudoconvex domain with a $C^{2}$-smooth boundary. Suppose that on $\bar{\Omega}$ we are given two smooth functions $\widetilde{\varphi}, \widetilde{\eta}$, where $\widetilde{\eta}>0$, whose Levi forms $\mathscr{L}_{\widetilde{\varphi}}$ and $\mathscr{L}_{\widetilde{\eta}}$ satisfy

$$
\widetilde{\eta} \mathscr{L}_{\widetilde{\varphi}}-\mathscr{L}_{\widetilde{\eta}} \geq Q+\frac{1}{4} \frac{\partial \widetilde{\eta} \otimes \overline{\partial \widetilde{\eta}}}{\widetilde{\eta}^{2}}
$$

with some positive hermitian form $Q$ on $\Omega$. Then, given a smooth $\bar{\partial}$-closed $(0,1)$-form $v=v_{1} d \bar{z}_{1}+\cdots+v_{n} d \bar{z}_{n}$ on $\Omega$ such that

$$
\mathscr{J}_{Q, \widetilde{\varphi}}(v):=\int_{\Omega}|v|_{Q}^{2} e^{-\widetilde{\varphi}} d^{2 n} z<\infty,
$$

one can solve the equation $\bar{\partial}\left(\sqrt{\widetilde{\eta}+\widetilde{\eta}^{2}} u\right)=v$ with a smooth function $u$ on $\Omega$ such that

$$
\int_{\Omega}|u|^{2} e^{-\widetilde{\varphi}} d^{2 n} z \leq 20 \mathscr{J}_{Q, \widetilde{\varphi}}(v) .
$$

Here $|v|_{Q}^{2}$ denotes the square of the length of $v$ with respect to the form $Q$ : If $\left(Q_{a \bar{b}}\right)_{a, b=1}^{n}$ is the coefficient matrix of $Q$ and $\left(Q^{a \bar{b}}\right)_{a, b=1}^{n}$ is its inverse matrix, then $|v|_{Q}^{2}=\sum_{a, b=1}^{n} Q^{a \bar{b}} v_{a} \bar{v}_{b}$.

Let $\left(D^{t}\right)_{r>t>0}$ be an exhaustion for $D$ by smooth bounded pseudoconvex domains $D^{t}$ such that $B\left(z^{0}, 2 r\right) \subset \subset D^{t}$ for each $t$.

CASE 1: $V$ is continuous. On each $D^{t}$ we can choose a regularization $V^{t}$ of $V$ such that $V \leq V^{t} \leq V+1 / 2 m$ on $D^{t}$. Then in the above lemma we choose $\Omega:=D^{t}$ and

$$
\widetilde{\varphi}_{t}(z)=|z|^{2}+2 m V^{t}(z)+n \log \left(t^{2}+\left|z-z^{0}\right|^{2}\right) .
$$

Next we put

$$
\widetilde{\eta}_{t}=\eta_{t}+\log \eta_{t}
$$

where

$$
\eta_{t}(z):=-\log \frac{r^{2}+t^{2}+\left|z-z^{0}\right|^{2}}{8 e R_{D}^{2}}
$$

and $R_{D}$ is the diameter of $D$. This function has values $>1$, and $-\eta_{t}$ and 
$-\widetilde{\eta}_{t}$ are plurisubharmonic on $D^{t}$. We estimate

$$
\begin{aligned}
\widetilde{\eta}_{t} \mathscr{L}_{\widetilde{\varphi}_{t}}-\mathscr{L}_{\widetilde{\eta}_{t}} & \geq \widetilde{\eta}_{t} \mathscr{L}_{|z|^{2}}-\left(1+\frac{1}{\eta_{t}}\right) \mathscr{L}_{\eta_{t}}+\frac{\partial \widetilde{\eta}_{t} \otimes \overline{\partial \widetilde{\eta}_{t}}}{\left(1+\eta_{t}\right)^{2}} \\
& \geq \widetilde{\eta}_{t} \mathscr{L}_{|z|^{2}}-\mathscr{L}_{\eta_{t}}+\frac{\partial \widetilde{\eta}_{t} \otimes \overline{\partial \widetilde{\eta}_{t}}}{4 \widetilde{\eta}_{t}^{2}},
\end{aligned}
$$

because $-\mathscr{L}_{\eta_{t}}$ is positive and $1+\eta_{t} \leq 2 \eta_{t} \leq 2 \widetilde{\eta}_{t}$. We will choose $Q:=$ $\widetilde{\eta}_{t} \mathscr{L}_{|z|^{2}}-\mathscr{L}_{\eta_{t}}$.

Our next aim is to estimate $|v|_{Q}$. On the support of $v$, which is contained in $B\left(z^{0}, r\right) \backslash B\left(z^{0}, r / 2\right)$, we have

$$
\begin{aligned}
Q & =\widetilde{\eta}_{t} \mathscr{L}_{|z|^{2}}-\mathscr{L}_{\eta_{t}} \geq \widetilde{\eta}_{t} \mathscr{L}_{|z|^{2}}+\frac{r^{2}+t^{2}}{\left(r^{2}+t^{2}+\left|z-z^{0}\right|^{2}\right)^{2}} \mathscr{L}_{\left|z-z^{0}\right|^{2}} \\
& \geq \mathscr{L}_{|z|^{2}}+\frac{\left|z-z^{0}\right|^{2}}{\left(r^{2}+t^{2}+\left|z-z^{0}\right|^{2}\right)^{2}} \mathscr{L}_{\left|z-z^{0}\right|^{2}} \\
& \geq \mathscr{L}_{|z|^{2}}+\frac{\partial\left(\left|z-z^{0}\right|^{2}\right) \otimes \overline{\partial\left(\left|z-z^{0}\right|^{2}\right)}}{\left(r^{2}+t^{2}+\left|z-z^{0}\right|^{2}\right)^{2}} \\
& \geq \widetilde{Q}:=\mathscr{L}_{|z|^{2}}+\frac{\partial\left(\left|z-z^{0}\right|^{2}\right)}{3 r^{2}} \otimes \frac{\overline{\partial\left(\left|z-z^{0}\right|^{2}\right)}}{3 r^{2}}
\end{aligned}
$$

which implies

$$
\begin{aligned}
|v|_{Q}^{2} & \leq|v|_{\widetilde{Q}}^{2} \leq \xi_{r}\left(\sup \left|\chi^{\prime}\right|\right)^{2}\left|\frac{\bar{\partial}\left|z-z^{0}\right|^{2}}{r^{2}}\right|_{\widetilde{Q}}=9 \xi_{r}\left(\sup \left|\chi^{\prime}\right|\right)^{2} \frac{\left|z-z^{0}\right|^{2}}{r^{4}+\left|z-z^{0}\right|^{2}} \\
& \leq 9 \xi_{r}\left(\sup \left|\chi^{\prime}\right|\right)^{2},
\end{aligned}
$$

where $\xi_{r}$ is the characteristic function of $B\left(z^{0}, r\right) \backslash B\left(z^{0}, r / 2\right)$. On the support of $v$ we also have

$$
\widetilde{\varphi}_{t}(z) \geq 2 m V(z)+2 n \log (r / 2) .
$$

This yields

$$
\mathscr{J}_{Q, \widetilde{\varphi_{t}}}(v) \leq 9\left(\sup \left|\chi^{\prime}\right|\right)^{2} \frac{4^{n}}{r^{2 n}} \int_{B\left(z^{0}, r\right)} e^{-2 m V} d^{2 n} z=c_{n} A\left[e^{-2 m V}, z^{0}, r\right],
$$

with $c_{n}=9 \cdot 4^{n}\left(\sup \left|\chi^{\prime}\right|\right)^{2}$ times the volume of the unit ball. By Lemma 2.2 we obtain a smooth solution $u_{t}$ to the equation $\bar{\partial}\left(\sqrt{\widetilde{\eta}_{t}+\widetilde{\eta}_{t}^{2}} u_{t}\right)=v$ such that

$$
\int_{D^{t}}\left|u_{t}\right|^{2} e^{-\widetilde{\varphi}_{t}} d^{2 n} z \leq 20 c_{n} A\left[e^{-2 m V}, z^{0}, r\right] .
$$

Next we observe that

$$
\begin{aligned}
\left(\widetilde{\eta}_{t}+\right. & \left.\widetilde{\eta}_{t}^{2}\right) e^{-2 m V} e^{\widetilde{\varphi}_{t}}=\left(\widetilde{\eta}_{t}+\widetilde{\eta}_{t}^{2}\right) e^{|z|^{2}+2 m\left(V^{t}-V\right)}\left(t^{2}+\left|z-z^{0}\right|^{2}\right)^{n} \\
& \leq 4 e^{\widetilde{R}_{D}^{2}+1} \max _{0<x \leq\left(2 R_{D}\right)^{2}}\left(x^{n}\left(\log \frac{8 e R_{D}^{2}}{r^{2}+x}+\left(\log \frac{8 e R_{D}^{2}}{r^{2}+x}\right)^{2}\right)\right) \leq C_{D}^{\prime}
\end{aligned}
$$


with

$$
\widetilde{R}_{D}:=\max _{z \in D}|z|, \quad C_{D}^{\prime}:=8 e^{\widetilde{R}_{D}^{2}+1}\left(2 R_{D}\right)^{n} \max _{0<\xi \leq 4}\left(\xi^{n}\left(\log \frac{4 e}{\xi}\right)^{2}\right) .
$$

The function

$$
f_{t}(z)=\chi\left(\frac{\left|z-z^{0}\right|^{2}}{r^{2}}\right)-\sqrt{\widetilde{\eta}_{t}+\widetilde{\eta}_{t}^{2}} u_{t}(z)
$$

now becomes an element of $\mathcal{H}_{2 m V}\left(D^{t}\right)$, with norm

$$
\left\|f_{t}\right\|_{2 m V, D^{t}} \leq\left(\gamma_{n} R_{D}^{n}+5 \sqrt{C_{D}^{\prime}}\right) \sqrt{A\left[e^{-2 m V}, z^{0}, r\right]},
$$

where $\gamma_{n}:=\sqrt{\text { volume of the unit ball }}$. To see this, note that

$$
\left\|\chi\left(\frac{\left|z-z^{0}\right|^{2}}{r^{2}}\right)\right\|_{2 m V, D^{t}}^{2} \leq \gamma_{n}^{2} r^{2 n} A\left[e^{-2 m V}, z^{0}, r\right]
$$

and $r \leq R_{D}$.

By a standard weak-star limit argument (similar to [17]) we find a holomorphic function $\widetilde{f}$ on $D$ of the form

$$
\widetilde{f}(z)=\chi\left(\frac{\left|z-z^{0}\right|^{2}}{r^{2}}\right)-\sqrt{\widetilde{\eta}+\widetilde{\eta}^{2}} u(z)
$$

where

$$
\widetilde{\eta}=\eta+\log \eta, \quad \eta=-\log \frac{r^{2}+\left|z-z^{0}\right|^{2}}{8 e R_{D}^{2}}
$$

that satisfies

$$
\|\tilde{f}\|_{2 m V, D} \leq\left(\gamma_{n} R_{D}^{n}+\sqrt{C_{D}^{\prime}}\right) \sqrt{A\left[e^{-2 m V}, z^{0}, r\right]} .
$$

Moreover,

$$
\int_{D}|u|^{2} e^{-\varphi} d^{2 n} z \leq 20 c_{n} A\left[e^{-2 m V}, z^{0}, r\right]
$$

with $\varphi=|z|^{2}+2 m V(z)+2 n \log \left|z-z^{0}\right|$. This gives $u\left(z^{0}\right)=0$. The function $\tilde{f} /\|\widetilde{f}\|_{2 m V}$ is a candidate for $K_{2 m V}\left(z^{0}\right)$. So we obtain

$$
K_{2 m V}\left(z^{0}, z^{0}\right) \geq \frac{\left|\widetilde{f}\left(z^{0}\right)\right|^{2}}{\|\widetilde{f}\|_{2 m V}^{2}} \geq \frac{C_{D}}{A\left[e^{-2 m V}, z^{0}, r\right]}
$$

with $C_{D}:=\left(\gamma_{n} R_{D}^{n}+5 \sqrt{C_{D}^{\prime}}\right)^{-2}$. From this the first claim follows immediately.

CASE 2: $V$ is arbitrary. First we fix a number $s \ll 1$ and consider the Demailly regularizations of the functions $V^{t}$, taken over the domain $D^{s}$. We will denote them by $\left(V^{t}\right)_{m, D^{s}}$. Here $t<s$. In explicit form, $\left(V^{t}\right)_{m, D^{s}}=$ $(2 m)^{-1} \log K_{2 m V^{t}, D^{s}}$, where

$$
K_{2 m V^{t}, D^{s}}(z)=\sup \left\{\left|f_{t}(z)\right|^{2} \mid f_{t} \in \mathcal{H}_{2 m V^{t}}\left(D^{s}\right),\left\|f_{t}\right\|_{2 m V^{t}} \leq 1\right\}
$$


on $D^{s}$. Our first claim is: There exists a null sequence $\left(t_{k}\right)_{k}$ such that $K_{2 m V^{t}, D^{s}}\left(z^{0}\right) \rightarrow K_{2 m V, D^{s}}\left(z^{0}\right):=\sup \left\{|f(z)|^{2} \mid f \in \mathcal{H}_{2 m V}\left(D^{s}\right),\|f\|_{2 m V} \leq 1\right\}$.

To see this we choose for each $t<s$ a function $f_{t, s} \in \mathcal{H}_{2 m V^{t}}\left(D^{s}\right)$ with $\left\|f_{t}\right\|_{2 m V^{t}} \leq 1$ such that

$$
K_{2 m V^{t}, D^{s}}\left(z^{0}\right)=\left|f_{t, s}\left(z^{0}\right)\right|^{2} .
$$

Then the Alaoglu-Bourbaki theorem can be applied to the functions $\widehat{f}_{t, s}:=$ $f_{t, s} e^{-m V^{t}}$, which belong to $L^{2}\left(D^{s}\right)$. We have $\left\|\widehat{f}_{t, s}\right\|_{L^{2}\left(D^{s}\right)} \leq 1$. We can choose a null sequence $\left(t_{k}\right)_{k}$ and a function $\widehat{f}_{0, s} \in L^{2}\left(D^{s}\right)$, having norm $\leq 1$ in $L^{2}\left(D^{s}\right)$, such that $\widehat{f}_{t_{k}, s} \rightarrow \widehat{f}_{0, s}$ in the weak-star topology in $L^{2}\left(D^{s}\right)$. The function $f_{0, s}:=\widehat{f}_{0, s} e^{-m V}$ is square-integrable with respect to the weighted Lebesgue measure $e^{-2 m V} d^{2 n} z$ over $D^{s}$ with $\left\|f_{0, s}\right\|_{2 m V} \leq 1$.

We claim that $f_{0, s}$ is even holomorphic. Since $V$ and $V^{t_{k}}$ are negative, the functions $e^{m V}, e^{m V^{t} k}$ are bounded. Therefore, the sequences $\left(f_{t_{k}, s}\right)_{k}$ and $\left(\widehat{f}_{t_{k}, s} e^{m V}\right)_{k}$ tend to $f_{0, s}$ in the weak-star topology of $L^{2}\left(D^{s}\right)$. If now $\beta$ denotes an arbitrary test form of bidegree $(0,1)$, and $\vartheta$ is the formal adjoint of $\bar{\partial}$ (in $\left.L^{2}\left(D^{s}\right)\right)$, then

$$
\begin{aligned}
\left\langle f_{0, s}, \vartheta \beta\right\rangle & =\lim _{k \rightarrow \infty}\left\langle\widehat{f}_{t_{k}, s} e^{m V}, \vartheta \beta\right\rangle=\lim _{k \rightarrow \infty}\left\langle f_{t_{k}, s}, e^{m\left(V-V^{t_{k}}\right)} \vartheta \beta\right\rangle \\
& =\lim _{k \rightarrow \infty}\left(\left\langle f_{t_{k}, s}, \vartheta \beta\right\rangle+\left\langle f_{t_{k}, s},\left(e^{m\left(V-V^{t_{k}}\right)}-1\right) \vartheta \beta\right\rangle\right) \\
& =\lim _{k \rightarrow \infty}\left\langle f_{t_{k}, s},\left(e^{m\left(V-V^{t_{k}}\right)}-1\right) \vartheta \beta\right\rangle .
\end{aligned}
$$

But

$$
\begin{aligned}
\left|\left\langle f_{t_{k}, s},\left(e^{m\left(V-V^{t_{k}}\right)}-1\right) \vartheta \beta\right\rangle\right| & =\left|\left\langle\widehat{f}_{t_{k}, s}, e^{m V^{t_{k}}}\left(e^{m\left(V-V^{t_{k}}\right)}-1\right) \vartheta \beta\right\rangle\right| \\
& \leq\left\|\widehat{f}_{t_{k}, s}\right\|_{L^{2}\left(D^{s}\right)}\left\|e^{m V^{t_{k}}}\left(e^{m\left(V-V^{t_{k}}\right)}-1\right) \vartheta \beta\right\|_{L^{2}\left(D^{s}\right)} \\
& \leq\left\|\left(e^{m\left(V-V^{t_{k}}\right)}-1\right) \vartheta \beta\right\|_{L^{2}\left(D^{s}\right)} \rightarrow 0
\end{aligned}
$$

as $k \rightarrow \infty$. This proves that $f_{0, s} \in \mathcal{H}_{2 m V}\left(D^{s}\right)$. It also follows that the functions $f_{t_{k}, s}$ tend pointwise to $f_{0, s}$. Hence we obtain the desired lower bound:

$$
\begin{aligned}
K_{2 m V, D^{s}}\left(z^{0}\right) & =\left|f_{0, s}\left(z^{0}\right)\right|^{2}=\lim _{k \rightarrow \infty}\left|f_{t_{k}, s}\left(z^{0}\right)\right|^{2} \geq C_{D^{s}} \lim _{k \rightarrow \infty} \frac{1}{A\left[e^{\left.-2 m V^{t_{k}}, z^{0}, r\right]}\right.} \\
& \geq C_{D^{s}} \frac{1}{A\left[e^{-2 m V}, z^{0}, r\right]} \geq C_{D} \frac{1}{A\left[e^{-2 m V}, z^{0}, r\right]} .
\end{aligned}
$$

Finally, we apply a similar weak-star limit argument to the functions

$$
f_{0, s}^{*}(z):= \begin{cases}f_{0, s} e^{-m V}(z) & \text { for } z \in D^{s} \\ 0 & \text { for } z \in D \backslash D^{s}\end{cases}
$$


to obtain a function $f_{0} \in \mathcal{H}_{2 m V}(D)$ with norm $\left\|f_{0}\right\|_{2 m V} \leq 1$ such that

$$
\left|f_{0}\left(z^{0}\right)\right|^{2} \geq \frac{C_{D}}{A\left[e^{-2 m V}, z^{0}, r\right]},
$$

proving part (a) of the lemma.

(b) The second assertion follows by taking the logarithm on both sides in (a).

We next estimate the integral

$$
\mathscr{J}_{m}(r):=\int_{B\left(z^{0}, r\right)} e^{-2 m V} d^{2 n} z
$$

for $r \in\left(0, \delta_{D}\left(z^{0}\right) / 16\right)$. Let $\mu_{V}$ be the measure defined by $\Delta V$.

Lemma 2.3. Let $z^{0}$ and $0<\varepsilon<\delta_{D}\left(z^{0}\right) / 4$ and $0<r<\varepsilon / 4$ as in the preceding lemma. If

$$
m \leq \frac{(2 \varepsilon)^{2 n-2}}{16(n+1) e \mu_{V}\left(B\left(z^{0}, 2 \varepsilon\right)\right)},
$$

then

$$
\mathscr{J}_{m}(r) \leq C_{n} \omega_{2 n} \varepsilon^{2 n} \exp \left(\frac{9 m}{8 c_{n}} \frac{\mu_{V}(B(0, \varepsilon))}{\varepsilon^{2 n-2}}-4^{n} \cdot 2 m M[V, 0, \varepsilon]\right)
$$

with some unimportant constant $C_{n}>0$. Here $\omega_{2 n}$ is the area of the unit sphere in $\mathbb{C}^{n}$.

Proof. Let us assume that $z^{0}=0$. We choose a number $\varepsilon \in\left(4 r, \delta_{D}(0)\right)$. By the Riesz representation theorem we can write

$$
V(z)=P_{\varepsilon}^{*}(z)+h_{\varepsilon}(z) \quad \text { for } z \in B(0, \varepsilon),
$$

where $P_{\varepsilon}^{*}$ is the Green potential,

$$
P_{\varepsilon}^{*}(z)=\int_{|\zeta|<\varepsilon} G_{\varepsilon}(z, \zeta) d \mu_{V}(\zeta),
$$

and $G_{\varepsilon}(z, \zeta)$ denotes the (real) Green function of $B(0, \varepsilon)$. Further, $h_{\varepsilon}$ is the smallest harmonic majorant for $V$ on $B(0, \varepsilon)$ and is given by the Poisson integral of $V$,

$$
h_{\varepsilon}(z)=\frac{1}{\omega_{2 n} \varepsilon} \int_{|\zeta|=\varepsilon} \frac{\varepsilon^{2}-|z|^{2}}{|z-\zeta|^{2 n}} V(\zeta) d S(\zeta) .
$$

The Green function for $n \geq 2$ is defined by

$$
G_{\varepsilon}(z, \zeta)= \begin{cases}E(z-\zeta)-E\left(\left(z^{*}-\zeta\right)|z| / \varepsilon\right) & \text { if } z \neq \zeta, z \neq 0, \\ E(\zeta)+\frac{1}{(2 n-2) \omega_{2 n}} \frac{1}{\varepsilon^{2 n-2}} & \text { if } z \neq \zeta, z=0, \\ -\infty & \text { if } z=\zeta .\end{cases}
$$


Here $z^{*}:=\varepsilon^{2} z /|z|^{2}$ and $E$ is the fundamental solution to the Laplacian,

$$
E(x):=\frac{-1}{(2 n-2) \omega_{2 n}} \frac{1}{|x|^{2 n-2}} .
$$

First we apply Harnack's inequality to the harmonic function $h_{\varepsilon}$. Note that $h_{\varepsilon} \leq 0$, because $V<0$. For $|z|<r$ we have

$$
\begin{aligned}
h_{\varepsilon}(z) & \geq \frac{1+|z| / \varepsilon}{(1-|z| / \varepsilon)^{2 n-1}} h_{\varepsilon}(0) \geq \frac{1+r / \varepsilon}{(1-r / \varepsilon)^{2 n-1}} h_{\varepsilon}(0) \\
& =\frac{1+r / \varepsilon}{(1-r / \varepsilon)^{2 n-1}} M[V, 0, \varepsilon] \geq 4^{n} M[V, 0, \varepsilon] .
\end{aligned}
$$

Furthermore, $G_{\varepsilon}(z, \zeta) \geq E(z-\zeta)$. Therefore, if we put

$$
P_{\varepsilon}(z):=\frac{-1}{(2 n-2) \omega_{2 n}} \int_{|\zeta|<\varepsilon} \frac{1}{|z-\zeta|^{2 n-2}} d \mu_{V}(\zeta),
$$

we obtain $P_{\varepsilon}^{*}(z) \geq P_{\varepsilon}(z)$, and altogether

$$
e^{-2 m V(z)} \leq e^{-4^{n} \cdot 2 m M[V, 0, \varepsilon]} e^{-2 m P_{\varepsilon}(z)} .
$$

This gives us

$$
\mathscr{J}_{m}(r) \leq e^{-4^{n} \cdot 2 m M[V, 0, \varepsilon]} \int_{|z|<r} e^{-2 m P_{\varepsilon}(z)} d^{2 n} z .
$$

Next we transform the term $P_{\varepsilon}(z)$ using ideas of [22] (see for instance p. 475). For $s \in(\varepsilon / 3, \varepsilon / 2)$ and $x \in \mathbb{C}^{n}$ with $|x|=s$ and $z \in B(0, r)$ we write

$$
\begin{aligned}
\left|P_{\varepsilon}(z)-P_{\varepsilon}(x)\right| & \leq|x-z| \int_{0}^{1}\left|\nabla P_{\varepsilon}(z+t(x-z))\right| d t \\
& \leq(r+s) \int_{0}^{1}\left|\nabla P_{\varepsilon}(z+t(x-z))\right| d t .
\end{aligned}
$$

Integrating over the sphere $\{|x|=s\}$ we find (note that $r \leq \varepsilon / 4 \leq 3 s / 4 \leq s$ )

$$
\begin{aligned}
\int_{|x|=s} P_{\varepsilon}(x) d S(x)- & I(s, z) \\
& \leq \omega_{2 n} s^{2 n-1} P_{\varepsilon}(z) \leq \int_{|x|=s} P_{\varepsilon}(x) d S(x)+I(s, z),
\end{aligned}
$$

where

$$
I(s, z):=2 s \int_{|x|=s} \int_{0}^{1}\left|\nabla P_{\varepsilon}(z+t(x-z))\right| d t d S(x) .
$$

To estimate the integral $I(s, z)$, we parametrize the positive hemisphere $M_{s}^{+}:=\left\{|\xi|=s \mid \xi_{2 n}>0\right\}$ by $\phi(\alpha):=\left(\alpha, \sqrt{s^{2}-|\alpha|^{2}}\right)$. Let $\psi(t, \alpha):=$ $z+t(\phi(\alpha)-z)$ for $(t, \alpha) \in(0,1) \times B_{2 n-1}(0, s)$. This defines an injective 
mapping from $(0,1) \times B_{2 n-1}(0, s)$ into $B_{2 n}(0,2 s)$. Its Jacobian determinant is

$$
\operatorname{det} J_{\psi}(t, \alpha)=-\frac{t^{2 n-1}}{\sqrt{s^{2}-|\alpha|^{2}}}\left(s^{2}-\langle\phi(\alpha), z\rangle\right)
$$

(where $\langle\cdot, \cdot\rangle$ denotes the euclidean inner product). Since $r<3 s / 4$, we obtain

$$
\left|\operatorname{det} J_{\psi}(t, \alpha)\right| \geq \frac{t^{2 n-1}\left(s^{2}-|\phi(\alpha)||z|\right)}{\sqrt{s^{2}-|\alpha|^{2}}} \geq \frac{t^{2 n-1} s^{2}}{4 \sqrt{s^{2}-|\alpha|^{2}}} .
$$

Then

$$
\begin{aligned}
\int_{M_{s}^{+}} \mid \nabla P_{\varepsilon}(z+t & (x-z)) \mid d S(x) \\
& =\int_{B_{2 n-1}(0, s)}\left|\nabla P_{\varepsilon}(z+t(\phi(\alpha)-z))\right| \frac{s}{\sqrt{s^{2}-|\alpha|^{2}}} d^{2 n-1} \alpha \\
& \leq \frac{4}{t^{2 n-1} s} \int_{B_{2 n-1}(0, s)}\left|\nabla P_{\varepsilon}(\psi(t, \alpha))\right|\left|\operatorname{det} J_{\psi}(t, \alpha)\right| d^{2 n-1} \alpha .
\end{aligned}
$$

Now we observe that

$$
|\psi(t, \alpha)-z|=t|\phi(\alpha)-z| \leq t(s+|z|) \leq 2 s t,
$$

which implies

$$
\frac{4}{t^{2 n-1} s} \leq 2^{2 n+1} s^{2 n-2} \frac{1}{|\psi(t, \alpha)-z|^{2 n-1}} .
$$

We obtain

$$
\begin{aligned}
\int_{0}^{1} \int_{M_{s}^{+}} \mid \nabla & P_{\varepsilon}(z+t(x-z)) \mid d S(x) d t \\
& \leq 4^{n} s^{2 n-2} \int_{(0,1) \times B_{2 n-1}(0, s)} \frac{\left|\nabla P_{\varepsilon}(\psi(t, \alpha))\right| \operatorname{det} J_{\psi}(t, \alpha)}{|\psi(t, \alpha)-z|^{2 n-1}} d^{2 n-1} \alpha d t \\
& \leq 4^{n} s^{2 n-2} \int_{B_{2 n}(0,2 s)} \frac{\left|\nabla P_{\varepsilon}(\zeta)\right|}{|z-\zeta|^{2 n-1}} d^{2 n} \zeta .
\end{aligned}
$$

A corresponding estimate holds for $\int_{0}^{1} \int_{M_{s}^{-}}\left|\nabla P_{\varepsilon}(z+t(x-z))\right| d S(x) d t$, where $M_{s}^{-}:=\left\{\xi \mid-\xi \in M_{s}^{+}\right\}$.

This proves that (note that $2 s \leq \varepsilon$ )

$$
I(s, z) \leq 4^{n+1} s^{2 n-1} \int_{B_{2 n}(0, \varepsilon)} \frac{\left|\nabla P_{\varepsilon}(\zeta)\right|}{|z-\zeta|^{2 n-1}} d^{2 n} \zeta .
$$

We now integrate over all $s \in(\varepsilon / 3, \varepsilon / 2)$ and divide by $\varepsilon^{2 n}$. This gives, in 
conjunction with (2.4),

$$
\begin{aligned}
& \frac{1}{c_{n} \varepsilon^{2 n}} \int_{\varepsilon / 3 \leq|x| \leq \varepsilon / 2} P_{\varepsilon}(x) d^{2 n} x-\mathscr{K}_{\varepsilon}(z) \\
& \leq P_{\varepsilon}(z) \leq \frac{1}{c_{n} \varepsilon^{2 n}} \int_{\varepsilon / 3 \leq|x| \leq \varepsilon / 2} P_{\varepsilon}(x) d^{2 n} x+\mathscr{K}_{\varepsilon}(z),
\end{aligned}
$$

where $c_{n}:=\frac{\omega_{2 n}}{2 n}\left((1 / 2)^{2 n}-(1 / 3)^{2 n}\right)$ and

$$
\mathscr{K}_{\varepsilon}(z)=\frac{4^{n+1}}{\omega_{2 n}} \int_{B_{2 n}(0, \varepsilon)} \frac{\left|\nabla P_{\varepsilon}(\zeta)\right|}{|z-\zeta|^{2 n-1}} d^{2 n} \zeta .
$$

We estimate

$$
\begin{aligned}
\int_{\varepsilon / 3 \leq|x| \leq \varepsilon / 2} P_{\varepsilon}(x) d^{2 n} x & =-\int_{\varepsilon / 3 \leq|x| \leq \varepsilon / 2}\left(\frac{1}{(2 n-2) \omega_{2 n}} \int_{|\zeta|<\varepsilon} \frac{d \mu_{V}(\zeta)}{|x-\zeta|^{2 n-2}}\right) d^{2 n} x \\
& =-\int_{|\zeta|<\varepsilon}\left(\frac{1}{(2 n-2) \omega_{2 n}} \int_{\varepsilon / 3 \leq|x| \leq \varepsilon / 2} \frac{d^{2 n} x}{|x-\zeta|^{2 n-2}}\right) d \mu_{V}(\zeta) \\
& \geq-\int_{|\zeta|<\varepsilon}\left(\frac{1}{(2 n-2) \omega_{2 n}} \int_{|x-\zeta| \leq 3 \varepsilon / 2} \frac{d^{2 n} x}{|x-\zeta|^{2 n-2}}\right) d \mu_{V}(\zeta) \\
& \geq-\frac{9}{16} \varepsilon^{2} \mu_{V}(B(0, \varepsilon))
\end{aligned}
$$

for $\varepsilon<1 / 2$. Thus we obtain from $(2.5)$, since $P_{\varepsilon} \leq 0$,

$$
-\frac{9}{16 c_{n} \varepsilon^{2 n-2}} \mu_{V}(B(0, \varepsilon))-\mathscr{K}_{\varepsilon}(z) \leq P_{\varepsilon}(z) \leq \mathscr{K}_{\varepsilon}(z)
$$

and

$$
\int_{|z|<r} e^{-2 m P_{\varepsilon}(z)} d^{2 n} z \leq \exp \left(\frac{9 \cdot 4 m}{16 c_{n} \varepsilon^{2 n-2}} \mu_{V}(B(0, \varepsilon))\right) \int_{|z|<r} e^{2 m \mathscr{K}_{\varepsilon}(z)} d^{2 n} z .
$$

We only have to estimate the integral

$$
\begin{aligned}
\int_{|z|<r} e^{2 m \mathscr{K}_{\varepsilon}(z)} d^{2 n} z= & \frac{1}{2 n} \omega_{2 n} r^{2 n}+2 m \int_{|z|<r} \mathscr{K}_{\varepsilon}(z) d^{2 n} z \\
& +\sum_{q=2}^{\infty} \frac{(2 m)^{q}}{q !}\left\|\mathscr{K}_{\varepsilon}\right\|_{L^{q}(B(0, r))}^{q} .
\end{aligned}
$$


First,

$$
\begin{aligned}
& \int_{B_{2 n}(0, \varepsilon)}\left|\nabla P_{\varepsilon}(\zeta)\right| d^{2 n} \zeta \leq \frac{1}{\omega_{2 n}} \int_{B_{2 n}(0, \varepsilon)}\left(\int_{B_{2 n}(0, \varepsilon)} \frac{d \mu_{V}(y)}{|\zeta-y|^{2 n-1}}\right) d^{2 n} \zeta \\
& =\frac{1}{\omega_{2 n}} \int_{B_{2 n}(0, \varepsilon)}\left(\int_{B_{2 n}(0, \varepsilon)} \frac{d^{2 n} \zeta}{|\zeta-y|^{2 n-1}}\right) d \mu_{V}(y) \leq(2 \varepsilon) \mu_{V}(B(0, \varepsilon)) .
\end{aligned}
$$

By Fubini's theorem we get

$$
\begin{aligned}
& 2 m \int_{|z|<r} \mathscr{K}_{\varepsilon}(z) d^{2 n} z=2 m \frac{4^{n+1}}{\omega_{2 n}} \int_{|z|<r} \int_{B_{2 n}(0, \varepsilon)} \frac{\left|\nabla P_{\varepsilon}(\zeta)\right|}{|z-\zeta|^{2 n-1}} d^{2 n} \zeta d^{2 n} z \\
& \quad=2 m \frac{4^{n+1}}{\omega_{2 n}} \int_{B_{2 n}(0, \varepsilon)}\left|\nabla P_{\varepsilon}(\zeta)\right|\left(\int_{|z|<r} \frac{1}{|z-\zeta|^{2 n-1}} d^{2 n} z\right) d^{2 n} \zeta \\
& \quad \leq(2 m)(2 \varepsilon) 4^{n+1} \int_{B_{2 n}(0, \varepsilon)}\left|\nabla P_{\varepsilon}(\zeta)\right| d^{2 n} \zeta \leq 4^{n+1} \cdot 2 m \cdot(2 \varepsilon)^{2} \mu_{V}(B(0, \varepsilon)),
\end{aligned}
$$

using (2.8). For $q \geq 2$ we estimate the norms $\left\|\mathscr{K}_{\varepsilon}\right\|_{L^{q}(B(0, r))}^{q}$ by means of Hölder's inequality (see also the proof of Theorem 1 in [22, p. 476]). We use the formula

$$
\left\|\mathscr{K}_{\varepsilon}\right\|_{L^{q}(B(0, r))} \sup _{F \in L^{p}(B(0, r)),\|F\|_{L^{p}(B(0, r))}=1}\left|\int_{B(0, r)} F(x) \mathscr{K}_{\varepsilon}(x) d^{2 n} x\right|
$$

(where $p=q /(q-1)$ ). Let $F \in L^{p}(B(0, r))$ be normalized. Then we get

$$
\left|\int_{B(0, r)} F(x) \mathscr{K}_{\varepsilon}(x) d^{2 n} x\right| \leq \int_{B(0, r) \times B(0, \varepsilon)} f(x, \zeta) g(x, \zeta) d^{2 n} x d^{2 n} \zeta
$$

with

$$
f(x, \zeta)=\left(\frac{\left|\nabla P_{\varepsilon}(\zeta)\right|}{|x-\zeta|^{2 n-1 / q}}\right)^{1 / q}, \quad g(x, \zeta)=F(x)\left(\frac{\left|\nabla P_{\varepsilon}(\zeta)\right|}{|x-\zeta|^{2 n-1-1 / q}}\right)^{1 / p} .
$$

The $L^{q}$-norm of $f$ is estimated by

$$
\begin{aligned}
\|f\|_{L^{q}(B(0, r) \times B(0, \varepsilon))}^{q} & =\int_{B(0, r) \times B(0, \varepsilon)} \frac{\left|\nabla P_{\varepsilon}(\zeta)\right|}{|x-\zeta|^{2 n-1 / q}} d^{2 n} x d^{2 n} \zeta \\
& =\int_{B(0, r)}\left(\frac{d^{2 n} x}{|x-\zeta|^{2 n-1 / q}}\right) \int_{B(0, \varepsilon)}\left|\nabla P_{\varepsilon}(\zeta)\right| d^{2 n} \zeta \\
& \leq \omega_{2 n} q(r+\varepsilon)^{1 / q} \int_{B(0, \varepsilon)}\left|\nabla P_{\varepsilon}(\zeta)\right| d^{2 n} \zeta \leq 4 \omega_{2 n} q \varepsilon^{1+1 / q} \mu_{V}(B(0, \varepsilon)),
\end{aligned}
$$

again by (2.8). 
We next consider the $L^{p}$-norm of $g$. Let

$$
u(t):=\int_{B(x, t)}\left|\nabla P_{\varepsilon}(\zeta)\right| d^{2 n} \zeta .
$$

First we observe that

$$
\begin{aligned}
\int_{B(0, \varepsilon)} \frac{\left|\nabla P_{\varepsilon}(\zeta)\right|}{|x-\zeta|^{2 n-1-1 / q}} d^{2 n} \zeta & \leq \int_{B(x, 2 \varepsilon)} \frac{\left|\nabla P_{\varepsilon}(\zeta)\right|}{|x-\zeta|^{2 n-1-1 / q}} d^{2 n} \zeta \\
& =\int_{0}^{2 \varepsilon}\left(\int_{|\zeta-x|=t} \frac{\left|\nabla P_{\varepsilon}(\zeta)\right|}{|x-\zeta|^{2 n-1-1 / q}} d S(\zeta)\right) d t \\
& =\int_{0}^{2 \varepsilon} \frac{1}{t^{2 n-1-1 / q}} u^{\prime}(t) d t \\
& =\frac{u(2 \varepsilon)}{(2 \varepsilon)^{2 n-1-1 / q}}+\left(2 n-1-\frac{1}{q}\right) \int_{0}^{2 \varepsilon} \frac{u(t)}{t^{2 n-1 / q}} d t .
\end{aligned}
$$

Now we note that

$$
u(t) \leq 2 t \mu_{V}(B(0, t)) \quad \text { and } \quad \frac{\mu_{V}(B(0, t))}{t^{2 n-2}} \leq \frac{\mu_{V}(B(0,2 \varepsilon))}{(2 \varepsilon)^{2 n-2}} .
$$

The second inequality follows from the fact that the function

$$
t \mapsto \mu_{V}(B(0, t)) / t^{2 n-2}
$$

is increasing (see [19, pp. 72-73]). Hence

$$
\int_{0}^{2 \varepsilon} \frac{u(t)}{t^{2 n-1 / q}} d t \leq 2 \frac{\mu_{V}(B(0,2 \varepsilon))}{(2 \varepsilon)^{2 n-2}} \int_{0}^{2 \varepsilon} \frac{d t}{t^{1-1 / q}}=2 q \varepsilon^{1 / q} \frac{\mu_{V}(B(0,2 \varepsilon))}{(2 \varepsilon)^{2 n-2}} .
$$

This implies

$$
\int_{B(0, \varepsilon)} \frac{\left|\nabla P_{\varepsilon}(\zeta)\right|}{|x-\zeta|^{2 n-1-1 / q}} d^{2 n} \zeta \leq 2(2 n+2) q \varepsilon^{1 / q} \frac{\mu_{V}(B(0,2 \varepsilon))}{(2 \varepsilon)^{2 n-2}} .
$$

Then

$$
\begin{aligned}
\|g\|_{L^{p}(B(0, r) \times B(0, \varepsilon))}^{p} & =\int_{B(0, r)}|F(x)|^{p}\left(\int_{B(0, \varepsilon)} \frac{\left|\nabla P_{\varepsilon}(\zeta)\right|}{|x-\zeta|^{2 n-1-1 / q}} d^{2 n} \zeta\right) d^{2 n} x \\
& \leq 2(2 n+2) q \varepsilon^{1 / q} \frac{\mu_{V}(B(0,2 \varepsilon))}{(2 \varepsilon)^{2 n-2}} \int_{B(0, r)}|F(x)|^{p} d^{2 n} x \\
& =2(2 n+2) q \varepsilon^{1 / q} \frac{\mu_{V}(B(0,2 \varepsilon))}{(2 \varepsilon)^{2 n-2}} .
\end{aligned}
$$


By Hölder's inequality we find (using $1 / p+1 / q=1$ ) that

$$
\begin{aligned}
\left|\int_{B(0, r)} F(x) \mathscr{K}_{\varepsilon}(x) d^{2 n} x\right| \leq & \left(4 \omega_{2 n} q \varepsilon^{1+1 / q} \mu_{V}(B(0, \varepsilon))\right)^{1 / q} \\
& \times\left(2(2 n+2) q \varepsilon^{1 / q} \frac{\mu_{V}(B(0,2 \varepsilon))}{(2 \varepsilon)^{2 n-2}}\right)^{1 / p} \\
\leq & (2 n+2)\left(2 \omega_{2 n}\right)^{1 / q} \cdot 2 q \mu_{V}(B(0,2 \varepsilon))(2 \varepsilon)^{2-2 n / p} \\
= & (2 n+2)\left(2 \omega_{2 n}\right)^{1 / q} \cdot 2 q \mu_{V}(B(0,2 \varepsilon))(2 \varepsilon)^{2-2 n+2 n / q} .
\end{aligned}
$$

This implies

$$
\left\|\mathscr{K}_{\varepsilon}\right\|_{L^{q}(B(0, r))}^{q} \leq 2 \omega_{2 n}(2 q)^{q}\left((2 n+2) \frac{\mu_{V}(B(0,2 \varepsilon))}{(2 \varepsilon)^{2 n-2}}\right)^{q}(2 \varepsilon)^{2 n} .
$$

Substituting this into (2.7) we obtain

$$
\begin{aligned}
& \int_{|z|<r} e^{2 m \mathscr{K}_{\varepsilon}(z)} d^{2 n} z \\
& \quad \leq \frac{1}{2 n} \omega_{2 n} r^{2 n}+4^{2 n+2} \omega_{2 n}(2 \varepsilon)^{2 n} \sum_{q=1}^{\infty} \frac{(2 q)^{q}}{q !}\left(\frac{8(n+1) m \mu_{V}(B(0,2 \varepsilon))}{(2 \varepsilon)^{2 n-2}}\right)^{q} \\
& \quad \leq C_{n} \omega_{2 n} \varepsilon^{2 n}
\end{aligned}
$$

if we choose

$$
m \leq \frac{(2 \varepsilon)^{2 n-2}}{16(n+1) e \mu_{V}(B(0,2 \varepsilon))}
$$

in order to make the above series converge.

In conjunction with (2.3) we get

$$
\begin{aligned}
\mathscr{J}_{m}(r) & \leq e^{-4^{n} \cdot 2 m M[V, 0, \varepsilon]} \int_{|z|<r} e^{-2 m P_{\varepsilon}(z)} d^{2 n} z \\
& \leq \exp \left(\frac{9 m}{8 c_{n}} \frac{\mu_{V}(B(0, \varepsilon))}{\varepsilon^{2 n-2}}-4^{n} \cdot 2 m M[V, 0, \varepsilon]\right) \int_{|z|<r} e^{2 m \mathscr{K}_{\varepsilon}(z)} d^{2 n} z \\
& \leq C_{n} \omega_{2 n} \varepsilon^{2 n} \exp \left(\frac{9 m}{8 c_{n}} \frac{\mu_{V}(B(0, \varepsilon))}{\varepsilon^{2 n-2}}-4^{n} \cdot 2 m M[V, 0, \varepsilon]\right) .
\end{aligned}
$$

This will give a lower bound for the regularization $V_{m}$ :

Lemma 2.4. Let $D$ be as above and $z^{0} \in D$. Let $\varepsilon<\delta_{D}\left(z^{0}\right) / 4$. If

$$
m \leq \frac{(2 \varepsilon)^{2 n-2}}{16(n+1) e \mu_{V}\left(B\left(z^{0}, 2 \varepsilon\right)\right)},
$$

then

$$
V_{m}\left(z^{0}\right) \geq-\frac{\log \left(C_{D} / C_{n}\right)}{2 m}-\frac{9}{16 c_{n}} \frac{\mu_{V}\left(B\left(z^{0}, \varepsilon\right)\right)}{\varepsilon^{2 n-2}}+4^{n} A\left[V, z^{0}, \varepsilon\right] .
$$


Proof. We combine Lemmas 2.1 and 2.3 with $r=\varepsilon / 8$ :

$$
\begin{aligned}
V_{m}\left(z^{0}\right) & \geq-\frac{\log C_{D}}{2 m}-\frac{1}{2 m} \log \left(A\left[e^{-2 m V}, z^{0}, \varepsilon / 8\right]\right) \\
& =-\frac{\log C_{D}}{2 m}-\frac{1}{2 m} \log \left(\frac{2 n 8^{2 n}}{\omega_{2 n} \varepsilon^{2 n}} \mathscr{J}_{m}(\varepsilon / 8)\right) \\
& \geq-\frac{\log \left(2 n C_{D} / C_{n}\right)}{2 m}-\frac{9}{16 c_{n}} \frac{\mu_{V}\left(B\left(z^{0}, \varepsilon\right)\right)}{\varepsilon^{2 n-2}}+4^{n} M\left[V, z^{0}, \varepsilon\right] .
\end{aligned}
$$

This gives the desired estimate, since $M\left[V, z^{0}, \varepsilon\right] \geq A\left[V, z^{0}, \varepsilon\right]$.

3. Application to the pluricomplex Green function. We start with a technical lemma:

Lemma 3.1. Assume that $D$ is bounded and hyperconvex and $\psi \in \operatorname{PSH}(D)$ is continuous, negative and satisfies $\|\psi\|_{\infty}=1$ and condition (1) of Main Theorem 1.1. Let $w, z^{0} \in D$. Then, for any $0<r<\frac{4}{5} \delta_{D}\left(z^{0}\right)$,

$$
\int_{B\left(z^{0}, 6 r / 5\right)}\left|\mathscr{G}_{D}(x, w)\right| d^{2 n} x \leq 2 \pi(n !)^{1 / n}|\psi(w)|^{1 / n} I\left(\frac{6}{5} r, h\right),
$$

and

$$
A\left[\left|\mathscr{G}_{D}(\cdot, w)\right|, z^{0}, r\right]+\frac{\mu_{\mathscr{G}_{D}(\cdot, w)}\left(B\left(z^{0}, r\right)\right)}{r^{2 n-2}} \leq \frac{C_{n}^{*}}{r^{2 n}}|\psi(w)|^{1 / n} I\left(\frac{6}{5} r, h\right)
$$

with some constant $C_{n}^{*}$ that depends only on $n$. Here we define, for $0<\varrho<$ $\delta_{D}\left(z^{0}\right)$,

$$
I(\varrho, h):=\left(\int_{B\left(z^{0}, \varrho\right)} h^{-1 /(n-1)} d^{2 n} x\right)^{1-1 / n} .
$$

Proof. We apply an idea from [7]. The key tool is an estimate obtained in [5]: Given an arbitrary bounded domain $D^{\prime}$ and negative locally bounded plurisubharmonic functions $u, v_{1}, \ldots, v_{n}$ on $D^{\prime}$ such that $\lim _{z \rightarrow q} u(z)=0$ for any $q \in \partial D^{\prime}$, we have

$$
\int_{D^{\prime}}|u|^{n} d d^{c} v_{1} \wedge \cdots \wedge d d^{c} v_{n} \leq n !\left\|v_{1}\right\|_{\infty} \cdot \ldots \cdot\left\|v_{n-1}\right\|_{\infty} \int_{D^{\prime}}\left|v_{n}\right|\left(d d^{c} u\right)^{n} .
$$

For an arbitrary number $L>1$ we put

$$
u_{L}:=\max \left\{\mathscr{G}_{D}(\cdot, w),-L\right\} .
$$

By the Hölder inequality we have 


$$
\begin{aligned}
& \int_{B\left(z^{0}, 6 r / 5\right)}\left|u_{L}\right| d^{2 n} x=\int_{B\left(z^{0}, 6 r / 5\right)}\left|u_{L}\right| h^{1 / n} \frac{1}{h^{1 / n}} d^{2 n} x \\
& \leq\left(\int_{B\left(z^{0}, 6 r / 5\right)}\left|u_{L}\right|^{n} h d^{2 n} x\right)^{1 / n}\left(\int_{B\left(z^{0}, 6 r / 5\right)} \frac{1}{h^{1 /(n-1)}} d^{2 n} x\right)^{1-1 / n} .
\end{aligned}
$$

We see that

$$
\int_{B\left(z^{0}, 6 r / 5\right)}\left|u_{L}\right|^{n} h d^{2 n} x \leq \int_{B\left(z^{0}, 6 r / 5\right)}\left|u_{L}\right|^{n}\left(d d^{c} \psi\right)^{n} .
$$

Now we can apply (3.3) for $D^{\prime}:=D$ and $v_{1}=\cdots=v_{n}=\psi$ to obtain (because of $\|\psi\|_{\infty}=1$ )

$$
\begin{aligned}
\int_{B\left(z^{0}, 6 r / 5\right)}\left|u_{L}\right|^{n} h d^{2 n} x & \leq \int_{D}\left|u_{L}\right|^{n}\left(d d^{c} \psi\right)^{n} \\
& \leq n ! \int_{D}|\psi|\left(d d^{c} \max \left\{\mathscr{G}_{D}(\cdot, w),-L\right\}\right)^{n} .
\end{aligned}
$$

We want to let $L$ tend to infinity. This is allowed, since the well-known convergence theorem of Bedford-Taylor gives that the currents

$$
T_{L}:=\left(d d^{c} \max \left\{\mathscr{G}_{D}(\cdot, w),-L\right\}\right)^{n}
$$

tend weakly to $(2 \pi)^{n}$ times the Dirac measure with center $w$ as $L \rightarrow \infty$. But, since all of them have the same total mass (namely $(2 \pi)^{n}$ ), we may apply Satz 45.7 of [1]. This gives us, in conjunction with the Beppo-Levi theorem, on letting $L \rightarrow \infty$ in (3.5),

$$
\int_{B\left(z^{0}, 6 r / 5\right)}\left|\mathscr{G}_{D}(x, w)\right|^{n} h d^{2 n} x \leq n ! \int_{D}|\psi|\left(d d^{c} \mathscr{G}_{D}(\cdot, w)\right)^{n}=(2 \pi)^{n} n !|\psi(w)|,
$$

and because of (3.4),

$$
\int_{B\left(z^{0}, 6 r / 5\right)}\left|\mathscr{G}_{D}(x, w)\right| d^{2 n} x \leq\left((2 \pi)^{n} n !|\psi(w)|\right)^{1 / n}\left(\int_{B\left(z^{0}, 6 r / 5\right)} h^{-1 /(n-1)} d^{2 n} x\right)^{1-1 / n} .
$$

From this we get (3.1).

Next we prove (3.2). This time we use (3.3) with $D^{\prime}:=B\left(z^{0}, 6 r / 5\right)$ and

$$
u(z)=\log \frac{1+\left|z-z^{0}\right|^{2} / 4 r^{2}}{2},
$$

and $v_{1}(z)=\cdots=v_{n-1}(z)=\left|z-z^{0}\right|^{2}-4 r^{2}, v_{n, L}(z)=\max \left\{\mathscr{G}_{D}(z, w),-L\right\}$ for a number $L>0$. Now (3.3) applies and gives, because of $|u| \geq \log (8 / 5)$ 
on $B\left(z^{0}, r\right)$,

$$
\begin{aligned}
& \left(\log \frac{8}{5}\right)^{n} \mu_{v_{n, L}}\left(B\left(z^{0}, r\right)\right) \\
& \leq \int_{B\left(z^{0}, r\right)}|u(z)|^{n} d \mu_{v_{n, L}}(z)=\int_{B\left(z^{0}, r\right)}|u(z)|^{n} d d^{\mathrm{c}} v_{1} \wedge \cdots \wedge d d^{\mathrm{c}} v_{n} \\
& \leq \int_{B\left(z^{0}, 6 r / 5\right)}|u(z)|^{n} d d^{\mathrm{c}} v_{1} \wedge \cdots \wedge d d^{\mathrm{c}} v_{n} \leq n !\left(4 r^{2}\right)^{n-1} \int_{B\left(z^{0}, 6 r / 5\right)}\left|v_{n, L}\right|\left(d d^{\mathrm{c}} u\right)^{n} \\
& \leq c_{n} \frac{1}{r^{2}} \int_{B\left(z^{0}, 6 r / 5\right)} \min \left\{\left|\mathscr{G}_{D}(x, w)\right|, L\right\} d^{2 n} x
\end{aligned}
$$

with some constant $c_{n}$. We let $L$ tend to infinity. By Beppo-Levi's theorem we obtain

$$
\frac{\mu_{\mathscr{G}_{D}(\cdot, w)}\left(B\left(z^{0}, r\right)\right)}{r^{2 n-2}} \leq \frac{c_{n}}{r^{2 n}(\log (8 / 5))^{n}} \int_{B\left(z^{0}, 6 r / 5\right)}\left|\mathscr{G}_{D}(x, w)\right| d^{2 n} x .
$$

From this and (3.1) the lemma follows.

We apply the above lemmas 2.4 and 3.1 to the case $V=\mathscr{G}_{D}(\cdot, w)$ for $w \in D$ and find

Lemma 3.2. Assume that $D$ is hyperconvex and $\psi \in \operatorname{PSH}(D)$ is continuous and satisfies $\|\psi\|_{\infty}=1$ and condition (1) from Main Theorem 1.1. Let $C_{n}^{*}$ be the constant from Lemma 3.1. Then, for any point $z^{0} \in D$ and any $0<\varepsilon<\delta_{D}\left(z_{0}\right) / 4$

$$
\left(\mathscr{G}_{D}(\cdot, w)\right)_{m}\left(z_{0}\right) \geq-\frac{1}{2 m} \log \frac{2 n C_{D}}{C_{n}}-\widetilde{C}_{n} C_{n}^{*} I_{0} \frac{|\psi(w)|^{1 / n}}{\varepsilon^{2 n}},
$$

provided that

$$
m \leq C_{n}^{* *} \frac{\varepsilon^{2 n}}{|\psi(w)|^{1 / n}}
$$

with the constants $C_{n}^{* *}:=1 / 16 e(n+1) C_{n}^{*} I_{0}, \widetilde{C}_{n}:=\left(9 / 16 c_{n}+4^{n}\right)$, and $I_{0}:=$ $\int_{D} h^{-1 /(n-1)}(x) d^{2 n} x$. The constant $c_{n}$ was defined after formula (2.5).

Proof. By Lemma 2.4 we have

$$
\begin{aligned}
\left(\mathscr{G}_{D}(\cdot, w)\right)_{m}\left(z^{0}\right) \geq & -\frac{1}{2 m} \log \frac{2 n C_{D}}{C_{n}} \\
& -\left(4^{n}\left|A\left[\mathscr{G}_{D}(\cdot, w), z^{0}, \varepsilon\right]\right|+\frac{9}{16 c_{n}} \frac{\mu \mathscr{G}_{D}(\cdot, w)\left(B\left(z^{0}, \varepsilon\right)\right)}{\varepsilon^{2 n-2}}\right),
\end{aligned}
$$

provided that

$$
m \leq \frac{(2 \varepsilon)^{2 n-2}}{16 e(n+1) \mu_{G_{D}(\cdot, w)}\left(B\left(z^{0}, 2 \varepsilon\right)\right)} .
$$


Now, estimate (3.2) of Lemma 3.1 with $r=\varepsilon$ gives us

$$
4^{n}\left|A\left[\mathscr{G}_{D}(\cdot, w), z^{0}, \varepsilon\right]\right|+\frac{9}{16 c_{n}} \frac{\mu_{\mathscr{G}_{D}(\cdot, w)}\left(B\left(z^{0}, \varepsilon\right)\right)}{\varepsilon^{2 n-2}} \leq \widetilde{C}_{n} C_{n}^{*} \frac{|\psi(w)|^{1 / n}}{(2 \varepsilon)^{2 n}} I_{0} .
$$

In particular,

$$
\frac{(2 \varepsilon)^{2 n-2}}{16 e(n+1) \mu_{\mathscr{G}_{D}(\cdot, w)}\left(B\left(z^{0}, 2 \varepsilon\right)\right)} \geq \frac{1}{16 e(n+1) C_{n}^{*} I_{0}} \frac{\varepsilon^{2 n}}{|\psi(w)|^{1 / n}} .
$$

This proves the lemma.

We want to apply the above results to the localization of the sublevel sets of the regularizations of $\mathscr{G}_{D}(\cdot, w)$.

A first step in this direction is

Lemma 3.3. Let $D \subset \subset \mathbb{C}^{n}$ be hyperconvex and $\psi$ as in Lemma 3.2. There exists a constant $r_{*}>0$, depending only on $n$, such that for $z^{0} \in D$ and $0<r<\min \left\{r_{*}, \delta_{D}\left(z^{0}\right)\right\}$,

$$
\sup _{x \in B\left(z^{0}, r\right)} \mathscr{G}_{D}(x, w) \geq-\frac{4^{2 n}}{C_{n}^{* *} \delta_{D}\left(z^{0}\right)^{2 n}}|\psi(w)|^{1 / n} \log \frac{C_{2}}{r^{n}}
$$

with the constants $C_{2}$ from (2.1) and $C_{n}^{* *}$ from Lemma 3.2 .

Proof. As in (2.1), for any $l>0$ we have

$$
\left(\mathscr{G}_{D}(\cdot, w)\right)_{l}\left(z^{0}\right) \leq-M(r)+\frac{1}{l} \log \frac{C_{2}}{r^{n}},
$$

where $M(r):=\left|\sup _{x \in B\left(z^{0}, r\right)} \mathscr{G}_{D}(x, w)\right|$. The number $C_{2}$ in (2.1) depends only on the dimension $n$. We assume $r<\sqrt[n]{C_{2}}$. Then the number

$$
l:=\frac{2 \log \left(C_{2} / r^{n}\right)}{M(r)} .
$$

is positive. This gives

$$
\left(\mathscr{G}_{D}(\cdot, w)\right)_{l}\left(z^{0}\right) \leq-\frac{1}{2} M(r)
$$

Further we put

$$
\varepsilon:=\left(\frac{l}{C_{n}^{* *}}|\psi(w)|^{1 / n}\right)^{1 / 2 n},
$$

where $C_{n}^{* *}$ is as in Lemma 3.2. Suppose that $\varepsilon<\delta_{D}\left(z^{0}\right) / 4$. Then Lemma 3.2 applies with $m=l$ and we obtain

$$
\left(\mathscr{G}_{D}(\cdot, w)\right)_{l}\left(z^{0}\right) \geq-\frac{1}{2 l} \log \frac{2 n C_{D}}{C_{n}}-\widetilde{C}_{n} C_{n}^{*} I_{0} \frac{|\psi(w)|^{1 / n}}{\varepsilon^{2 n}}=-\frac{C_{n}^{\prime}}{l}
$$

with

$$
C_{n}^{\prime}:=\log \frac{2 n C_{D}}{C_{n}}+C_{n}^{* *} \widetilde{C}_{n} C_{n}^{*} I_{0}
$$


Combining this with (3.6) we find

$$
-\frac{1}{2} M(r) \geq-\frac{C_{n}^{\prime}}{l}=-C_{n}^{\prime} \frac{M(r)}{2 \log \left(C_{2} / r^{n}\right)},
$$

Hence we would have, for $0<r<r_{*}$,

$$
1 \leq \frac{C_{n}^{\prime}}{\log \left(C_{2} / r^{n}\right)} \leq \frac{C_{n}^{\prime}}{\log \left(C_{2} / r_{*}^{n}\right)}<1,
$$

provided we choose $r_{*}$ suitably; the latter is possible uniformly in $z^{0}, w$. This contradiction implies

$$
\frac{\log \left(C_{2} / r^{n}\right)}{C_{n}^{* *} M(r)}|\psi(w)|^{1 / n}=\frac{l}{2 C_{n}^{* *}}|\psi(w)|^{1 / n}=2 \varepsilon^{2 n} \geq\left(\delta_{D}\left(z^{0}\right) / 4\right)^{2 n} .
$$

This means that

$$
M(r) \leq\left(\frac{4}{\delta_{D}\left(z^{0}\right)}\right)^{2 n} \frac{\log \left(C_{2} / r^{n}\right)}{C_{n}^{* *}}|\psi(w)|^{1 / n},
$$

which proves the lemma.

The same method allows a growth estimate in the spirit of the Hopf lemma:

Lemma 3.4. Suppose that $\psi: D \rightarrow[-1,0)$ is a smooth plurisubharmonic exhaustion function for D with property (1) of Main Theorem 1.1. Then there exists a constant $\gamma_{1}>0$ such that

$$
\psi \leq-\gamma_{1} \delta_{D}^{2 n^{2}}
$$

Proof. Let $w \in D$. We choose

$$
\varepsilon:=\left(\frac{3 n}{C_{n}^{* *}}\right)^{1 / 2 n}|\psi(w)|^{1 / 2 n^{2}},
$$

where $C_{n}^{* *}$ is the constant from Lemma 3.2. For any point $z \in D$ such that $\delta_{D}(z)>4 \varepsilon$, Lemma 3.2 applies with $m=3 n$. Hence

$$
\begin{aligned}
\left(\mathscr{G}_{D}(\cdot, w)\right)_{m}(z) & \geq-\frac{1}{6 n} \log \frac{2 n C_{D}}{C_{n}}-C_{n}^{\prime} \frac{|\psi(w)|^{1 / n}}{\varepsilon^{2 n}} \\
& =-\frac{1}{6 n} \log \frac{2 n C_{D}}{C_{n}}-\frac{C_{n}^{\prime} C_{n}^{* *}}{3 n} .
\end{aligned}
$$

Since $m>2 n$, the function $\left(\mathscr{G}_{D}(\cdot, w)\right)_{m}$ has a pole at $w$, which implies

$$
\delta_{D}(w) \leq 4 \varepsilon=4\left(\frac{3 n C_{n}^{\prime}}{C_{n}^{* *}}\right)^{1 / 2 n}|\psi(w)|^{1 / 2 n^{2}} .
$$

From this the claim follows with $\gamma_{1}:=4^{-2 n^{2}}\left(C_{n}^{* *} / 3 n C_{n}^{\prime}\right)^{n}$. 
Lemma 3.5. If $D$ and $\psi$ are as in the preceding lemma, then

$$
\mathscr{G}_{D}(z, w) \geq \frac{\log \left(2 R_{D} / \delta_{D}(w)\right)}{\inf _{B\left(w, \delta_{D}(w) / 2\right)}|\psi|} \cdot \psi(z)
$$

and in particular

$$
\mathscr{G}_{D}(z, w) \geq \gamma_{2} \frac{\psi(z)}{\delta_{D}(w)^{2 n^{2}+1}}
$$

for any $z \in D$ such that $|z-w| \geq \delta_{D}(w) / 2$, where $\gamma_{2}:=2^{2 n^{2}+1} R_{D} / \gamma_{1}$.

Proof. We proceed as in the proof of Lemma 2.4 of [12]. Let $v$ be the following function:

$$
v(x)= \begin{cases}\max \left\{C \psi(x), \log \frac{|x-w|}{R_{D}}\right\} & \text { if }|x-w| \geq \delta_{D}(w) / 2, \\ \log \frac{|x-w|}{R_{D}} & \text { if }|x-w| \leq \delta_{D}(w) / 2 .\end{cases}
$$

The lemma will be proved if $v$ is well-defined. For this the constant $C>0$ must be chosen in such a way that

$$
C \psi(x) \leq \log \frac{\delta_{D}(w)}{2 R_{D}} \quad \text { on } \partial B\left(w, \delta_{D}(w) / 2\right) .
$$

This estimate is satisfied, if we choose

$$
C \geq \frac{\log \left(2 R_{D} / \delta_{D}(w)\right)}{\inf _{B\left(w, \delta_{D}(w) / 2\right)}|\psi|}
$$

This proves (a).

For the proof of (b) we observe that from Lemma 3.4 we know $\psi(x) \leq$ $-\gamma_{1} \delta_{D}(x)^{2 n^{2}}$ for all $x \in D$. Since $\delta_{D}(x) \geq \delta_{D}(w) / 2$ on $\partial B\left(w, \delta_{D}(w) / 2\right)$, this gives

$$
|\psi(x)| \geq 2^{-2 n^{2}} \gamma_{1} \delta_{D}(w)^{2 n^{2}} \quad \text { on } \partial B\left(w, \delta_{D}(w) / 2\right) .
$$

Hence we choose

$$
C:=\frac{2^{2 n^{2}+1} R_{D}}{\gamma_{1} \delta_{D}(w)^{2 n^{2}+1}}, \quad \gamma_{2}:=\frac{2^{2 n^{2}+1} R_{D}}{\gamma_{1}} .
$$

In both cases (a) and (b), on $D \backslash B\left(w, \delta_{D}(w) / 2\right)$ we get

$$
\mathscr{G}_{D}(z, w) \geq v(z) \geq C \psi(z)=\gamma_{2} \frac{\psi(z)}{\delta_{D}(w)^{2 n^{2}+1}} .
$$

4. On the boundary behavior of the pluricomplex Green function. First we make two general observations. 
Lemma 4.1. Let $D$ be a bounded domain in $\mathbb{C}^{n}$. For any $m>1$ and $w, z \in D$,

$$
K_{2 m \mathscr{G}_{D}(\cdot, w)}(z, z) \geq\left(\frac{|z-w|}{n R_{D}}\right)^{2 m+2} K_{D}(z, z)
$$

and hence

$$
\left(\mathscr{G}_{D}(\cdot, w)\right)_{m}(z) \geq\left(1+\frac{1}{m}\right) \log \frac{|z-w|}{n R_{D}}+\frac{1}{2 m} \log K_{D}(z, z) .
$$

Proof. Given $z \in D$ we choose $j$ such that $\left|z_{j}-w_{j}\right| \geq|z-w| / n$. For $m>1$ let $[m]$ denote the integer part of $m$. If now $f \in H^{2}(D)$ is arbitrary with $\|f\|=1$, then the function

$$
f_{j}(x):=\left(\frac{x_{j}-w_{j}}{R_{D}}\right)^{[m]+1} f(x)
$$

belongs to $\mathcal{H}_{2 m \mathscr{G}_{D}(\cdot, w)}(D)$, since (note that $\mathscr{G}_{D}(x, w) \geq \log \frac{|x-w|}{R_{D}}$ )

$$
\begin{aligned}
\left|f_{j}(x)\right|^{2} e^{-2 m \mathscr{G}_{D}(x, w)} & \leq\left|f_{j}(x)\right|^{2}\left(\frac{R_{D}}{|x-w|}\right)^{2 m} \\
& \leq\left(\frac{|x-w|}{R_{D}}\right)^{2([m]+1)-2 m}|f(x)|^{2} \leq|f(x)|^{2} .
\end{aligned}
$$

This implies (if we choose $f=K_{D}(\cdot, z) / \sqrt{K_{D}(z, z)}$ )

$$
\begin{aligned}
& K_{2 m \mathscr{G}_{D}(\cdot, w)}(z, z) \geq \frac{\left|f_{j}(z)\right|^{2}}{\left\|f_{j}\right\|_{2 m \mathscr{G}_{D}(\cdot, w)}^{2}} \geq\left|f_{j}(z)\right|^{2} \\
& \geq\left(\frac{|z-w|}{n R_{D}}\right)^{2 m+2} K_{D}(z, z) .
\end{aligned}
$$

Taking log on both sides and dividing by $2 m$ we obtain the lemma.

Next we estimate the modulus of continuity of the Demailly regularization $\left(\mathscr{G}_{D}(\cdot, w)\right)_{m}$ as follows:

Lemma 4.2. Suppose that $D_{1} \subset \subset \mathbb{C}^{n}$ is any bounded domain. Then there is a constant $C_{0}^{\prime}>0$ such that, for any $m>0$ and $z^{0}, w \in D_{1}$ with $\delta_{D_{1}}\left(z^{0}\right) \geq$ $4 \delta_{D_{1}}(w)$,

$$
\left|\left(\mathscr{G}_{D_{1}}(\cdot, w)\right)_{m}\left(z_{*}\right)-\left(\mathscr{G}_{D_{1}}(\cdot, w)\right)_{m}\left(z^{0}\right)\right| \leq C_{0}^{\prime} \frac{1}{m}\left(\frac{2 n R_{D_{1}}}{\delta_{D}\left(z^{0}\right)}\right)^{m+1} \frac{\left|z_{*}-z^{0}\right|}{\delta_{D_{1}}\left(z^{0}\right)^{n+1}}
$$

whenever $z_{*} \in B\left(z^{0}, \delta_{D_{1}}\left(z^{0}\right) / 8\right)$. 
Proof. Fix $l \in\{1, \ldots, n\}$. Then we have

$$
\begin{aligned}
\left|\frac{\partial}{\partial z_{l}}\left(\mathscr{G}_{D_{1}}(\cdot, w)\right)_{m}(z)\right| & =\frac{1}{2 m} \frac{\left|\frac{\partial}{\partial z_{l}} K_{2 m \mathscr{G}_{D_{1}}(\cdot, w)}(z, z)\right|}{K_{2 m \mathscr{G}_{D_{1}}(\cdot, w)}(z, z)} \\
& \leq \frac{1}{2 m} \frac{\left|\frac{\partial^{2}}{\partial z_{l} \partial \bar{z}_{l}} K_{2 m \mathscr{G}_{D_{1}}(\cdot, w)}(z, z)\right|^{1 / 2}}{\sqrt{K_{2 m \mathscr{S}_{D_{1}}(\cdot, w)}(z, z)}} .
\end{aligned}
$$

The second inequality is due to the logarithmic plurisubharmonicity of $K_{2 m \mathscr{G}_{D_{1}}(\cdot, w)}(z, z)$. From Bergman theory we know that

$$
\frac{\partial^{2}}{\partial z_{l} \partial \bar{z}_{l}} K_{2 m \mathscr{G}_{D_{1}}(\cdot, w)}(z, z)=\sup _{f \in \mathcal{H}\left(2 m \mathscr{G}_{D_{1}}(\cdot, w)\right),\|f\|_{2 m \mathscr{G}_{D_{1}}(\cdot, w)}=1}\left|\frac{\partial f}{\partial z_{l}}(z)\right|^{2},
$$

from which it follows that

$$
\left|\frac{\partial}{\partial z_{l}}\left(\mathscr{G}_{D_{1}}(\cdot, w)\right)_{m}(z)\right| \leq \frac{1}{2 m} \frac{\sup _{f \in \mathcal{H}\left(2 m \mathscr{G}_{D_{1}}(\cdot, w)\right),\|f\|_{2 m \mathscr{G}_{D_{1}}(\cdot, w)}=1}\left|\frac{\partial f}{\partial z_{l}}(z)\right|}{\sqrt{K_{2 m \mathscr{G}_{D_{1}}}(z, w)}} .
$$

Now, any function $f \in \mathcal{H}\left(2 m \mathscr{G}_{D_{1}}(\cdot, w)\right)$ belongs to $H^{2}\left(D_{1}\right)$. Hence

$$
\left|\frac{\partial f}{\partial z_{l}}(z)\right| \leq c_{n} \frac{\|f\|_{L^{2}\left(D_{1}\right)}}{\delta_{D_{1}}(z)^{n+1}} \leq c_{n} \frac{\|f\|_{2 m \mathscr{G}_{D_{1}(\cdot, w)}}}{\delta_{D_{1}}(z)^{n+1}}
$$

for any $z \in D_{1}$. Furthermore,

$$
K_{2 m \mathscr{G}_{D_{1}}(z, w)}(z, z) \geq\left(\frac{|z-w|}{n R_{D_{1}}}\right)^{2 m+2} K_{D_{1}}(z, z) \geq \frac{1}{\operatorname{vol}\left(D_{1}\right)}\left(\frac{|z-w|}{n R_{D_{1}}}\right)^{2 m+2} .
$$

This will give

$$
\begin{aligned}
\left|\frac{\partial}{\partial z_{l}}\left(\mathscr{G}_{D_{1}}(\cdot, w)\right)_{m}(z)\right| & \leq \frac{1}{2 m} \frac{\sup _{f \in \mathcal{H}\left(2 m \mathscr{G}_{D_{1}}(\cdot, w)\right),\|f\|_{2 m \mathscr{G}_{D_{1}}(\cdot, w)} \mid}\left|\frac{\partial f}{\partial z_{l}}(z)\right|}{\sqrt{K_{2 m \mathscr{G}_{D_{1}}(\cdot, w)}(z, z)}} \\
& \leq \frac{\sqrt{\operatorname{vol}\left(D_{1}\right)}}{2 m}\left(\frac{n R_{D_{1}}}{|z-w|}\right)^{m+1} \delta_{D_{1}}(z)^{-(n+1)} \\
& \leq \frac{\sqrt{\operatorname{vol}\left(D_{1}\right)}}{2 m}\left(\frac{2 n R_{D_{1}}}{\delta_{D_{1}}\left(z^{0}\right)}\right)^{m+1} \delta_{D_{1}}(z)^{-(n+1)}
\end{aligned}
$$

on $B\left(z^{0}, \delta_{D_{1}}\left(z^{0}\right) / 8\right)$. From this the assertion follows by the mean value theorem.

In the next step we prove a quantitative result on upper semicontinuity of the Green function.

Lemma 4.3. Assume that $D$ and $\psi$ are as in Theorem 1.1 and $\gamma_{2}$ is the constant from Lemma 3.5. Then there are constants $\delta_{0}, \widetilde{C}_{1}, \widetilde{C}_{2}>0$ such 
that, for any $z^{0}, w \in D$ with $\delta_{D}(w)<\min \left\{\delta_{0}, \delta_{D}(z) / 4\right\}$ and $\mathscr{G}_{D}\left(z^{0}, w\right) \leq$ $-\gamma_{2} \delta_{D}(w)$, we have

$$
\mathscr{G}_{D}\left(z_{*}, w\right) \leq \mathscr{G}_{D}\left(z^{0}, w\right)+\widetilde{C}_{1} \delta_{D}(w)+\widetilde{C}_{2} \frac{|\psi(w)|^{1 / 3 n}}{\delta_{D}\left(z^{0}\right)^{n+3}}
$$

whenever $\left|z_{*}-z^{0}\right| \leq \exp \left(-1 /|\psi(w)|^{1 / 2 n}\right)$.

Proof. Let $m>n$. We define the function

$$
v(x):= \begin{cases}\mathscr{G}_{D}(x, w) & \text { if } \mathscr{G}_{D}(x, w) \geq-\gamma_{2} \delta_{D}(w), \\ \max \left\{\mathscr{G}_{D}(x, w), \widetilde{v}_{w}(x)\right\} & \text { if } \mathscr{G}_{D}(x, w) \leq-\gamma_{2} \delta_{D}(w) .\end{cases}
$$

Let $C_{2}$ be the constant from the proof of Lemma 3.3. We define the function $\widetilde{v}_{w}$ by

$$
\widetilde{v}_{w}(x):=\left(\mathscr{G}_{D}(\cdot, w)\right)_{m}(x)-\frac{1}{m} \log \frac{C_{2}}{r_{w}^{n}}-\gamma_{2} \delta_{D}(w) .
$$

If the radius $r_{w}$ is less than or equal to the boundary distance of the set $S:=\left\{x \in d \mid \mathscr{G}_{D}(x, w)=-\gamma_{2} \delta_{D}(w)\right\}$, the function $v$ is well-defined and negative. We have

$$
v \leq(1-n / m) \mathscr{G}_{D}(\cdot, w) .
$$

Next we want to estimate the boundary distance of $S$ from below, using the growth condition on $\psi$.

Let $x \in S$ with $\delta_{D}(x)<1$. If $|x-w| \leq \delta_{D}(w) / 2$, we get $\delta_{D}(x) \geq \delta_{D}(w) / 2$, hence

$$
\log \frac{1}{\delta_{D}(x)} \leq \log \frac{2}{\delta_{D}(w)}
$$

If $|x-w| \geq \delta_{D}(w) / 2$, we find, because of Lemma 3.5,

$$
-\gamma_{2} \delta_{D}(w)=\mathscr{G}_{D}(x, w) \geq \gamma_{2} \frac{\psi(x)}{\delta_{D}(w)^{2 n^{2}+1}}
$$

hence

$$
\delta_{D}(w)^{2 n^{2}+2} \leq|\psi(x)| \leq \widehat{C}_{1} \exp \left(-\widehat{C}_{2}\left(\log \frac{1}{\delta_{D}(x)}\right)^{\alpha}\right) .
$$

For $w \in D$ with $\delta_{D}(w)<\delta_{0}:=\min \left\{1 / 2, \widehat{C}_{1}^{-1 /\left(2 n^{2}+2\right)}, \widehat{C}_{1}^{1 /\left(2 n^{2}+2\right)}\right\}$ this leads to

$$
\log \frac{1}{\delta_{D}(x)} \leq\left(\frac{1}{\widehat{C}_{2}} \log \frac{\widehat{C}_{1}}{\delta_{D}(w)^{2 n^{2}+2}}\right)^{1 / \alpha}
$$

and hence

$$
\delta_{D}(x) \geq \exp \left(-\left(\frac{1}{\widehat{C}_{2}} \log \frac{\widehat{C}_{1}}{\delta_{D}(w)^{2 n^{2}+2}}\right)^{1 / \alpha}\right) .
$$

This estimate holds trivially for $x \in S$ with $\delta_{D}(x) \geq 1$. 
Hence we may choose

$$
r_{w}=\min \left\{\delta_{D}(w) / 2, \exp \left(-\left(\frac{1}{\widehat{C}_{2}} \log \frac{\widehat{C}_{1}}{\delta_{D}(w)^{2 n^{2}+2}}\right)^{1 / \alpha}\right)\right\} .
$$

We want to estimate the term $\log \left(C_{2} / r_{w}^{n}\right)$, which appeared in the definition of $\widetilde{v}_{w}$, when $\delta_{D}(w)<\delta_{0}$.

From $|\psi(w)| \leq \widehat{C}_{1} \exp \left(-\widehat{C}_{2} \log \left(1 / \delta_{D}(w)\right)^{\alpha}\right)$ we obtain

$$
\log \frac{1}{\delta_{D}(w)} \leq\left(\frac{1}{\widehat{C}_{2}} \log \frac{\widehat{C}_{1}}{|\psi(w)|}\right)^{1 / \alpha}
$$

Assume now that $r_{w}=\delta_{D}(w) / 2$. Then

$$
\begin{aligned}
\log \frac{C_{2}}{r_{w}^{n}} & =\log C_{2}+n \log \frac{2}{\delta_{D}(w)} \\
& \leq \log C_{2}+2 n \log \frac{1}{\delta_{D}(w)} \\
& \leq \log C_{2}+2 n\left(\frac{1}{\widehat{C}_{2}} \log \frac{\widehat{C}_{2}}{|\psi(w)|}\right)^{1 / \alpha} .
\end{aligned}
$$

If

$$
r_{w}=\exp \left(-\left(\frac{1}{\widehat{C}_{2}} \log \frac{\widehat{C}_{1}}{\delta_{D}(w)^{2 n^{2}+2}}\right)^{1 / \alpha}\right)
$$

we get

$$
\begin{aligned}
\log \frac{C_{2}}{r_{w}^{n}} & =\log C_{2}+n \log \frac{1}{r_{w}} \\
& =\log C_{2}+n\left(\frac{1}{\widehat{C}_{2}} \log \frac{\widehat{C}_{2}}{\delta_{D}(w)^{2 n^{2}+2}}\right)^{1 / \alpha} \\
& \leq \log C_{2}+n\left(\frac{2}{\widehat{C}_{2}}\right)^{1 / \alpha}\left(\log \frac{1}{\delta_{D}(w)^{2 n^{2}+2}}\right)^{1 / \alpha} \\
& \leq \log C_{2}+n\left(\frac{2 n^{2}+2}{\widehat{C}_{2}}\right)^{2 / \alpha^{2}}\left(\log \frac{\widehat{C}_{2}}{|\psi(w)|}\right)^{1 / \alpha^{2}} .
\end{aligned}
$$

Let $z^{0}, w \in D$ be as in the hypothesis of the lemma. If $z_{*} \in D$ is close enough to $z^{0}$, we can estimate, for any $m>0$,

$$
\begin{aligned}
\mathscr{G}_{D}\left(z_{*}, w\right) \leq & \left(\mathscr{G}_{D}(\cdot, w)\right)_{m}\left(z_{*}\right)+\frac{1}{C_{1} m} \quad(\text { by }(2.1)) \\
= & \left(\mathscr{G}_{D}(\cdot, w)\right)_{m}\left(z^{0}\right) \\
& +\left(\mathscr{G}_{D}(\cdot, w)\right)_{m}\left(z_{*}\right)-\left(\mathscr{G}_{D}(\cdot, w)\right)_{m}\left(z^{0}\right)+\frac{1}{C_{1} m} .
\end{aligned}
$$


But $\mathscr{G}_{D}\left(z^{0}, w\right) \leq-\gamma_{2} \delta_{D}(w)$, hence

$$
\begin{aligned}
\left(\mathscr{G}_{D}(\cdot, w)\right)_{m}\left(z^{0}\right) \leq & \widetilde{v}_{w}\left(z^{0}\right)+\frac{1}{m} \log \frac{C_{2}}{r_{w}^{n}}+\gamma_{2} \delta_{D}(w) \\
\leq & v\left(z^{0}\right)+\frac{1}{m} \log \frac{C_{2}}{r_{w}^{n}}+\gamma_{2} \delta_{D}(w) \\
\leq & \left(1-\frac{n}{m}\right) \mathscr{G}_{D}\left(z^{0}, w\right)+\frac{1}{m} \log \frac{C_{2}}{r_{w}^{n}}+\gamma_{2} \delta_{D}(w) \\
\leq & \left(1-\frac{n}{m}\right) \mathscr{G}_{D}\left(z^{0}, w\right)+\frac{\log C_{2}}{m}+\gamma_{2} \delta_{D}(w) \\
& +\frac{n}{m}\left(\frac{2 n^{2}+2}{\widehat{C}_{2}}\right)^{2 / \alpha^{2}}\left(\log \frac{\widehat{C}_{1}}{|\psi(w)|}\right)^{1 / \alpha^{2}}
\end{aligned}
$$

and

$$
\left|\left(\mathscr{G}_{D}(\cdot, w)\right)_{m}\left(z_{*}\right)-\left(\mathscr{G}_{D}(\cdot, w)\right)_{m}\left(z^{0}\right)\right| \leq C_{0}^{\prime} \frac{1}{m}\left(\frac{2 n R_{D}}{\delta_{D}\left(z^{0}\right)}\right)^{m+1} \frac{\left|z_{*}-z^{0}\right|}{\delta_{D}\left(z^{0}\right)^{n+1}}
$$

if $\left|z_{*}-z^{0}\right|<\delta_{D}\left(z^{0}\right) / 8$. We choose

$$
m:=\frac{1}{\log \left(2 n R_{D} / \delta_{D}\left(z^{0}\right)\right)} \frac{1}{|\psi(w)|^{1 / 2 n}} .
$$

Then for $z_{*} \in D$ such that $\left|z_{*}-z^{0}\right| \leq \exp \left(-1 /|\psi(w)|^{1 / 2 n}\right)$ we estimate

$$
\begin{aligned}
C_{0}^{\prime} \frac{1}{m}\left(\frac{2 n R_{D}}{\delta_{D}\left(z^{0}\right)}\right)^{m+1} \frac{\left|z_{*}-z^{0}\right|}{\delta_{D}\left(z^{0}\right)^{n+1}} & \leq C_{0}^{\prime} \frac{1}{m} \exp \left(\frac{1}{|\psi(w)|^{1 / 2 n}}\right) \frac{\left|z_{*}-z^{0}\right|}{\delta_{D}\left(z^{0}\right)^{n+1}} \\
& \leq C_{0}^{\prime} \frac{1}{m} \frac{1}{\delta_{D}\left(z^{0}\right)^{n+1}} .
\end{aligned}
$$

For this we note that, after shrinking $\delta_{0}$ if necessary, for $\delta_{D}(w) \leq \delta_{D}\left(z^{0}\right) / 4$ we have

$$
\exp \left(-\frac{1}{|\psi(w)|^{1 / 2 n}}\right) \leq \delta_{D}(w) / 2 \leq \delta_{D}\left(z^{0}\right) / 8
$$

We combine (4.1) and (4.2) and the estimate

$$
-\frac{n}{m} \mathscr{G}_{D}\left(z^{0}, w\right) \leq \frac{n}{m} \log \frac{R_{D}}{\left|z^{0}-w\right|} \leq \frac{n}{m} \log \frac{2 R_{D}}{\delta_{D}\left(z^{0}\right)} .
$$

This in conjunction with our choice of $m$ gives, for $z^{0}, w \in D$ as in the hypothesis of the lemma and $\delta_{D}(w) \leq \delta_{0}$,

$$
\begin{aligned}
\mathscr{G}_{D}\left(z_{*}, w\right) \leq & \mathscr{G}_{D}\left(z^{0}, w\right)+C_{3} \frac{\log \left(2 n R_{D} / \delta_{D}\left(z^{0}\right)\right)}{\delta_{D}\left(z^{0}\right)^{n+2}}|\psi(w)|^{1 / 2 n}(\log (1 /|\psi(w)|))^{1 / \alpha^{2}} \\
& +\gamma_{2} \delta_{D}(w) .
\end{aligned}
$$

From this we obtain the lemma. 
Proof of Theorem 1.1. Let $\delta_{0}>0$ be as in the preceding lemma. Let $K \subset D$ be compact; without loss of generality, let $\delta_{D}(K)<1$. Assume that $w \in D$ is such that $\delta_{D}(w) \leq \min \left\{\delta_{0}, \delta_{D}(K) / 4\right\}$ and $A:=\sup _{z \in K}\left|\mathscr{G}_{D}(z, w)\right|$. Then there exists a $z^{0} \in K$ such that $\mathscr{G}_{D}\left(z^{0}, w\right)=-A$. We put $r:=$ $\exp \left(-1 /|\psi(w)|^{1 / 2 n}\right)$. Assume that $A \geq \gamma_{2} \delta_{D}(w)$. Then, combining Lemmas 3.3 and 4.3 , we obtain

$$
\begin{aligned}
-A=\mathscr{G}_{D}\left(z^{0}, w\right) & \geq-\frac{\widetilde{C}_{n}}{\delta_{D}\left(z^{0}\right)^{2 n}}|\psi(w)|^{1 / n} \log \frac{C_{2}}{r^{n}}-\widetilde{C}_{1} \delta_{D}(w)-\widetilde{C}_{2} \frac{|\psi(w)|^{1 / 3 n}}{\delta_{D}\left(z^{0}\right)^{n+3}} \\
& \geq-C^{*} \frac{|\psi(w)|^{1 / 3 n}}{\delta_{D}\left(z^{0}\right)^{2 n+3}}-\widetilde{C}_{1} \delta_{D}(w) \\
& \geq-C^{*} \frac{|\psi(w)|^{1 / 3 n}}{\delta_{D}(K)^{2 n+3}}-\widetilde{C}_{1} \delta_{D}(w)
\end{aligned}
$$

with some universal constant $C^{*}$. The case $A \leq \gamma_{2} \delta_{D}(w)$ is trivial. This implies the theorem.

5. Estimation of the Bergman distance. We are now going to prove Theorem 1.4. The proof is based on the methods developed in [14].

Using Theorem 1.1 we localize the sublevel sets of the Green function.

Lemma 5.1. Under the hypotheses of Theorem 1.4, there are constants $0<C_{2}^{\prime} \leq 1 \leq C_{1}^{\prime}, C_{3}^{\prime}$ such that for any $w \in D$ sufficiently close to $\partial D$, $\left\{z \in D \mid \mathscr{G}_{D}(z, w) \leq-1\right\}$ $\subset\left\{\left.z \in D\left|C_{2}^{\prime} \exp \left(-C_{3}^{\prime}\left(\log \frac{1}{|\psi(w)|}\right)^{1 / \alpha^{2}}\right) \leq \delta_{D}(z) \leq C_{1}^{\prime}\right| \psi(w)\right|^{1 / 3 n(2 n+1)}\right\}$.

Proof. Suppose $z \in D$ and $\mathscr{G}_{D}(z, w) \leq-1$.

CASE 1: $\delta_{D}(w) / 4 \leq \delta_{D}(z) \leq 4 \delta_{D}(w)$. Then, because

$$
C_{2}^{\prime} \exp \left(-C_{3}^{\prime}\left(\log \frac{1}{|\psi(w)|}\right)^{1 / \alpha}\right) \leq \delta_{D}(w)
$$

and $\delta_{D}(w) \leq \gamma_{1}^{-1 / 2 n^{2}}|\psi(w)|^{1 / 2 n^{2}} \leq \gamma_{1}^{-1 / 2 n^{2}}|\psi(w)|^{1 / 3 n(2 n+1)} \quad$ (for $w$ close to $\partial D)$, as follows from our hypothesis on the growth of $|\psi|$ and from Lemma 3.4, respectively, we obtain

$$
C_{2}^{\prime} \exp \left(-C_{3}^{\prime}\left(\log \frac{1}{|\psi(w)|}\right)^{1 / \alpha}\right) \leq \delta_{D}(z) \leq 4 \gamma_{1}^{-1 / 2 n^{2}}|\psi(w)|^{1 / 3 n(2 n+1)} .
$$

CASE 2: $\delta_{D}(z) \geq 4 \delta_{D}(w)$. First, Theorem 1.1 with $K=\{z\}$ gives $\delta_{D}(z) \leq \widetilde{C}^{1 /(2 n+1)}|\psi(w)|^{1 / 3 n(2 n+1)}$. For the lower bound on $\delta_{D}(z)$ we note that for $z$ with $\delta_{D}(z)<1$ Lemma 3.5 implies, in conjunction with condition 
(2) of Main Theorem 1.1,

$$
1 \leq\left|\mathscr{G}_{D}(z, w)\right| \leq \gamma_{2} \frac{|\psi(z)|}{\delta_{D}(w)^{2 n^{2}+1}} \leq \gamma_{2} \widehat{C}_{1} \frac{\exp \left(-\widehat{C}_{2}\left|\log \delta_{D}(z)\right|^{\alpha}\right)}{\delta_{D}(w)^{2 n^{2}+1}} .
$$

This implies

$$
\delta_{D}(z) \geq \exp \left(-\widehat{C}_{2}^{-1 / \alpha}\left(\log \frac{\gamma_{2}}{\widehat{C}_{1} \delta_{D}(w)^{2 n^{2}+1}}\right)^{1 / \alpha}\right) .
$$

We apply the growth condition on $\psi$, this time at the point $w$, to find

$$
\log \frac{1}{\delta_{D}(w)} \leq\left(\frac{1}{\widehat{C}_{2}} \log \frac{\widehat{C}_{1}}{|\psi(w)|}\right)^{1 / \alpha}
$$

Combining this with the preceding estimate we obtain

$$
\delta_{D}(z) \geq C_{2}^{\prime} \exp \left(-C_{3}^{\prime}\left(\log \frac{1}{|\psi(w)|}\right)^{1 / \alpha^{2}}\right)
$$

with a suitable constant $C_{2}^{\prime} \leq 1$. If $\delta_{D}(z) \geq 1$, this estimate holds trivially.

CASE 3: $\delta_{D}(z) \leq \delta_{D}(w) / 4$. Again we have $|z-w| \geq \delta_{D}(w) / 2$, hence the lower bound on $\delta_{D}(z)$ follows as in Case 2. The upper bound on $\delta_{D}(z)$ is obtained as in Case 1.

The above lemma enables us to estimate the Bergman distance between two points that have different boundary distances.

LEMMA 5.2. In the situation of Theorem 1.4 there exists a constant $c_{0}>0$ such that for $A, B \in D$ one has $d_{D}^{\mathrm{B}}(A, B) \geq c_{0}$ provided that

$$
C_{2}^{\prime} \exp \left(-C_{3}^{\prime}\left(\log \frac{1}{|\psi(A)|}\right)^{1 / \alpha^{2}}\right) \geq C_{1}^{\prime}|\psi(B)|^{1 / 3 n(2 n+1)},
$$

where $C_{1}^{\prime}, C_{2}^{\prime}, C_{3}^{\prime}$ are as in Lemma 5.1.

Proof. Under the hypothesis of the lemma, the sublevel sets $\left\{\mathscr{G}_{D}(\cdot, A)\right.$ $<-1\}$ and $\left\{\mathscr{G}_{D}(\cdot, B)<-1\right\}$ are disjoint. Hence by Theorem 4.4 of [4],

$$
d_{D}^{\mathrm{B}}(A, B) \geq c_{0}:=\frac{\pi}{2}-\arctan \left(1+\frac{4 e^{n}}{\eta_{n}}\right),
$$

with $\eta_{n}=\int_{n}^{\infty} \frac{d x}{x e^{x}}$.

Proof of Theorem 1.4. Let $0<\tau_{0}<e^{-e}$ be so small that $\sup |\psi| \geq 2 \tau_{0}$ and

$$
C_{2}^{\prime} \exp \left(-C_{3}^{\prime}\left(\log \frac{1}{x}\right)^{1 / \alpha^{2}}\right) \leq \frac{1}{2} C_{1}^{\prime} x^{1 / 3 n(2 n+1)} \quad \text { for all } 0<x \leq \tau_{0}
$$


We first consider a smooth curve $\Gamma:[0, L] \rightarrow D$ such that $|\psi(\Gamma(0))|=\tau_{0}$ and $|\psi(\Gamma(L))|<\tau_{0}$. Furthermore, we suppose that

$$
|\psi(\Gamma(L))|^{1 / 3 n(2 n+1)}<\min \left\{\tau_{0}^{1 / 3 n(2 n+1)}, \frac{C_{2}^{\prime}}{C_{1}^{\prime}} e^{C_{3}^{\prime}\left|\log \tau_{0}\right|^{1 / \alpha^{2}}}\right\} .
$$

We put

$$
\varphi(t):=C_{2}^{\prime} \exp \left(-C_{3}^{\prime}\left(\log \frac{1}{|\psi(\Gamma(t))|}\right)^{1 / \alpha^{2}}\right)
$$

Then

$$
C_{1}^{\prime}|\psi(\Gamma(L))|^{1 / 3 n(2 n+1)}<\varphi(0)<C_{1}^{\prime}|\psi(\Gamma(0))|^{1 / 3 n(2 n+1)} .
$$

The left inequality comes from $(\dagger \dagger)$ and the right from $(\dagger)$. Hence we find $t_{1} \in(0, L)$ such that

$$
C_{1}^{\prime}\left|\psi\left(\Gamma\left(t_{1}\right)\right)\right|^{1 / 3 n(2 n+1)}=\varphi(0) .
$$

We choose a sequence $0=t_{0}<t_{1}<\cdots<t_{\nu}<L$ of maximal length such that

$$
\varphi\left(t_{s}\right)=C_{1}^{\prime}\left|\psi\left(\Gamma\left(t_{s+1}\right)\right)\right|^{1 / 3 n(2 n+1)}, \quad 0 \leq s<\nu .
$$

Then we obtain

$$
\operatorname{Bergman} \operatorname{length}(\Gamma) \geq \sum_{s=0}^{\nu-1} \operatorname{Bergman} \operatorname{length}\left(\Gamma \mid\left[t_{s}, t_{s+1}\right]\right) \geq c_{0} \nu
$$

with $c_{0}$ as in Lemma 5.2.

Next we estimate $\nu$ from below. By (5.1) we have

Also,

$$
\log \frac{1}{\left|\psi\left(\Gamma\left(t_{s+1}\right)\right)\right|}=3 n(2 n+1) \log \frac{C_{1}^{\prime}}{\varphi\left(t_{s}\right)} .
$$

$$
\log \frac{1}{|\psi(\Gamma(t))|}=\left(\frac{1}{C_{3}^{\prime}} \log \frac{C_{2}^{\prime}}{\varphi(t)}\right)^{\alpha^{2}}
$$

which gives

$$
\left(\frac{1}{C_{3}^{\prime}} \log \frac{C_{2}^{\prime}}{\varphi\left(t_{s+1}\right)}\right)^{\alpha^{2}}=3 n(2 n+1) \log \frac{C_{1}^{\prime}}{\varphi\left(t_{s}\right)} .
$$

We write

$$
a_{s}:=\frac{1}{C_{3}^{\prime}} \log \frac{C_{1}^{\prime}}{\varphi\left(t_{s}\right)}
$$

and deduce from (5.3) that

$$
\left(-C_{4}^{\prime}+a_{s+1}\right)^{\alpha^{2}}=3 n(2 n+1) C_{3}^{\prime} a_{s}
$$

with $C_{4}^{\prime}:=\frac{1}{C_{3}^{\prime}} \log \frac{C_{1}^{\prime}}{C_{2}^{\prime}}$. Note that $C_{4}^{\prime}>0$. In this way we obtain the recursive formula

$$
a_{s+1}=C_{5}^{\prime} a_{s}^{1 / \alpha^{2}}+C_{4}^{\prime}
$$


with $C_{5}^{\prime}:=\left(3 n(2 n+1) C_{3}^{\prime}\right)^{1 / \alpha^{2}}$. Hence the sequence $\left(a_{s}\right)_{s}$ increases, and

$$
a_{s+1} \leq C_{6}^{\prime} a_{s}^{1 / \alpha^{2}}
$$

where the constant $C_{6}^{\prime}:=1+C_{5}^{\prime}+C_{4}^{\prime} a_{0}^{-1 / \alpha^{2}}$ depends only on $\tau_{0}$.

By induction on $l$ we get

$$
a_{l} \leq C_{6}^{\prime 1+\alpha^{-2}+\cdots+\alpha^{-2(l-1)}} a_{0}^{\alpha^{-2 l}} .
$$

Note that

$$
a_{0}=\frac{1}{C_{3}^{\prime}} \log \frac{C_{1}^{\prime}}{\varphi(0)} \geq \frac{1}{C_{3}^{\prime}} \log \frac{C_{1}^{\prime}}{C_{1}^{\prime} \tau_{0}^{1 / 3 n(2 n+1)}} \geq 2
$$

after shrinking $\tau_{0}$ again. Also, $a_{s}>1$ for all $s \geq 0$.

We choose $l=\nu$ and take logarithms on both sides:

$$
\begin{aligned}
\log a_{\nu} & \leq\left(1+\alpha^{-2}+\cdots+\alpha^{-2(\nu-1)}\right) \log C_{6}^{\prime}+\alpha^{-2 \nu} \log a_{0} \\
& =\frac{\alpha^{-2 \nu}-1}{\alpha^{-2}-1} \log C_{6}^{\prime}+\alpha^{-2 \nu} \log a_{0} \leq C_{7}^{\prime} \alpha^{-2 \nu}
\end{aligned}
$$

with $C_{7}^{\prime}:=\frac{\alpha^{2}\left(1-\alpha^{2 \nu}\right)}{1-\alpha^{2}} \log C_{6}^{\prime}+\log a_{0}$. Finally, we get

$$
\nu \geq C_{8}^{\prime} \log \log a_{\nu}-\frac{\log C_{7}^{\prime}}{\log (1 / \alpha)},
$$

with $C_{8}^{\prime}:=\frac{1}{2 \log (1 / \alpha)}$.

We now estimate $a_{\nu}$ from below as follows: Assume that

$$
\varphi\left(t_{\nu}\right)>C_{1}^{\prime}|\psi(\Gamma(L))|^{1 / 3 n(2 n+1)} .
$$

Then we would obtain

$$
C_{1}^{\prime}|\psi(\Gamma(L))|^{1 / 3 n(2 n+1)}<\varphi\left(t_{\nu}\right)<C_{1}^{\prime}\left|\psi\left(\Gamma\left(t_{\nu}\right)\right)\right|^{1 / 3 n(2 n+1)},
$$

the right inequality being implied by $(\dagger)$. In particular, we could choose a number $t_{\nu+1} \in\left(t_{\nu}, L\right)$ such that

$$
C_{1}^{\prime}\left|\psi\left(\Gamma\left(t_{\nu+1}\right)\right)\right|^{1 / 3 n(2 n+1)}=\varphi\left(t_{\nu}\right),
$$

which is (5.1) for $s=\nu$, a contradiction to the maximality of $\nu$.

Thus we have $\varphi\left(t_{\nu}\right) \leq C_{1}^{\prime}|\psi(\Gamma(L))|^{1 / 3 n(2 n+1)}$ and consequently

$$
a_{\nu}=\frac{1}{C_{3}^{\prime}} \log \frac{C_{1}^{\prime}}{\varphi\left(t_{\nu}\right)} \geq \frac{1}{3 n(2 n+1) C_{3}^{\prime}} \log \frac{1}{|\psi(\Gamma(L))|} .
$$

Combining this with (5.4) and (5.2) we obtain

$$
\text { Bergman length }(\Gamma) \geq \log \log \log (1 /|\psi(\Gamma(L))|)-C_{9} .
$$

The right-hand side is well-defined, since $|\psi(\Gamma(L))|<\tau_{0}<e^{-e}$. 
Finally, we fix $P \in D$ such that $|\psi(P)|>\tau_{0}$, and $Q \in D$ close enough to the boundary of $D$ such that

$$
C_{1}|\psi(Q)|^{1 / 3 n(2 n+1)} \leq C_{2}^{\prime} \exp \left(-C_{3}^{\prime}\left(\log \frac{1}{\tau_{0}}\right)^{1 / \alpha^{2}}\right) .
$$

Then there exists a geodesic $\widetilde{\Gamma}$ in the Bergman metric with length $d_{D}^{\mathrm{B}}(P, Q)$. It contains a piece $\Gamma:[0, L] \rightarrow D$ such that $\Gamma(L)=Q$, to which the preceding considerations apply. Its length satisfies

$$
d_{D}^{\mathrm{B}}(P, Q) \geq \log \log \log (1 /|\psi(\Gamma(L))|)-C_{9}=\log \log \log (1 /|\psi(Q)|)-C_{9} .
$$

This gives the desired result.

6. Supplementary remarks and proof of Theorem 1.3. We want to discuss condition (1) of Main Theorem 1.1. A negative plurisubharmonic function $\psi$ with property (2) of that theorem whose reciprocal is integrable induces a function $\psi_{1}$ that has both properties (1) and (2). We will prove this as follows.

Lemma 6.1. Assume that $\psi: D \rightarrow[-1,0)$ is plurisubharmonic and continuous. Then the following statements are equivalent:

(a) There exists an exponent $\eta>0$ such that $|\psi|^{-\eta}$ is integrable over $D$.

(b) There exists an exponent $N$ and a constant $\gamma_{*}$ such that $\psi \leq-\gamma_{*} \delta_{D}^{N}$.

Proof. The implication (b) $\Rightarrow(\mathrm{a})$ is clear.

For the proof of the reverse implication we use Lemma 6.2 below. If $\eta>0$ is sufficiently small, then for a suitable choice of $L>0$ we can apply Lemma 3.4 to the function

$$
\psi_{1}(z):=-\left(-\psi(z) e^{-L|z|^{2}}\right)^{\eta},
$$

since

$$
\left(d d^{\mathrm{c}} \psi_{1}\right)^{n} \geq(\eta L / 2)^{n}\left|\psi_{1}\right|^{n}\left(d d^{\mathrm{c}}|z|^{2}\right)^{n} .
$$

Now $\psi_{1}$, and hence also $\psi$, satisfies an estimate of the form $\psi \leq-\gamma_{*} \delta_{D}^{N}$.

We complete the proof of the above lemma by proving

Lemma 6.2. Let $\psi$ be a continuous negative plurisubharmonic function on a domain $D_{1} \subset \subset \mathbb{C}^{n}$. Then, given a number $\eta \in(0,1)$, one can choose $L>0$ in such a way that the function $\psi_{1}(z):=-\left(-\psi(z) e^{-L|z|^{2}}\right)^{\eta}$ is also plurisubharmonic, and satisfies

$$
\left(d d^{\mathrm{c}} \psi_{1}\right)^{n} \geq(\eta L / 2)^{n}\left|\psi_{1}\right|^{n}\left(d d^{\mathrm{c}}|z|^{2}\right)^{n} .
$$

Proof. Because the desired estimate is meant in the sense of distributions, it is enough to show it over an arbitrary subdomain $D^{\prime} \subset \subset D$. 
First we assume that $\psi$ is of class $C^{\infty}$. We repeat the computation from [11] to find

$$
\begin{aligned}
\mathscr{L}_{\psi_{1}}(z ; X) & =\eta\left(-\psi(z) e^{-L|z|^{2}}\right)^{\eta-1} e^{-L|z|^{2}}\left(\mathscr{L}_{\psi}(z ; X)+L|\psi(z)||X|^{2}\right. \\
- & \left.2 L \eta \operatorname{Re}\langle\partial \psi(z), X\rangle\langle z, X\rangle+\frac{1-\eta}{-\psi(z)}|\langle\partial \psi(z), X\rangle|^{2}-\eta L^{2}|\langle z, X\rangle|^{2}\right) .
\end{aligned}
$$

But

$-2 L \eta \operatorname{Re}\langle\partial \psi(z), X\rangle\langle z, X\rangle+\frac{1-\eta}{-\psi(z)}|\langle\partial \psi(z), X\rangle|^{2} \geq-\frac{\eta^{2} L^{2}}{1-\eta}|\psi(z)||\langle z, X\rangle|^{2}$.

This gives

$$
\begin{aligned}
\mathscr{L}_{\psi_{1}}(z ; X)= & \eta\left(-\psi(z) e^{-L|z|^{2}}\right)^{\eta-1} e^{-L|z|^{2}} \\
& \times\left(\mathscr{L}_{\psi}(z ; X)+L|\psi(z)|\left(|X|^{2}-\frac{\eta L}{1-\eta}|\langle z, X\rangle|^{2}\right)\right) .
\end{aligned}
$$

Given $\eta$ we let $L$ be so small that $\eta L|z|^{2} /(1-\eta)<1 / 2$ throughout $D$. Then

$$
\begin{aligned}
\mathscr{L}_{\psi_{1}}(z ; X) & \geq \eta\left(-\psi(z) e^{-L|z|^{2}}\right)^{\eta-1} e^{-L|z|^{2}}\left(\mathscr{L}_{\psi}(z ; X)+\frac{L}{2}|\psi(z)||X|^{2}\right) \\
& \geq \frac{\eta L}{2}\left|\psi_{1}(z)\right||X|^{2} .
\end{aligned}
$$

Taking the determinants we obtain

$$
\left(d d^{\mathrm{c}} \psi_{1}\right)^{n} \geq(\eta L / 2)^{n}\left|\psi_{1}\right|^{n}\left(d d^{\mathrm{c}}|z|^{2}\right)^{n} .
$$

If $\psi$ is not necessarily smooth, we approximate $\psi$ from above on $D^{\prime}$ by a sequence $\left(v_{j}\right)_{j}$ which decreases to $\psi$ and apply the first part of the proof. The numbers $\eta, L$ do not depend on the $v_{j}$ 's or on $D^{\prime}$. Then we get

$$
\left(d d^{\mathrm{c}}-\left(-v_{j} e^{-L|z|^{2}}\right)^{\eta}\right)^{n} \geq(\eta L / 2)^{n}\left(-v_{j} e^{-L|z|^{2}}\right)^{n \eta}\left(d d^{\mathrm{c}}|z|^{2}\right)^{n} .
$$

Now Bedford-Taylor's approximation theorem for the Monge-Ampère operator gives the claim.

This enables us to replace (roughly speaking) condition (1) of Main Theorem 1.1 by the condition that $1 /|\psi|$ is integrable over $D$.

COROLlary. Assume that the continuous plurisubharmonic function $\psi$ is negative on $D$ and $1 /|\psi|$ is integrable over $D$. Then $\psi_{1}:=-(-\psi)^{1-1 / n}$ is also negative and plurisubharmonic, and

$$
\left(d d^{\mathrm{c}} \psi_{1}\right)^{n} \geq \gamma_{3}|\psi|^{n-1}\left(d d^{\mathrm{c}}|z|^{2}\right)^{n} .
$$

If , furthermore, $\psi$ is smooth and satisfies also condition (2) of Main Theorem 1.1, then $\psi_{1}$ satisfies both conditions (1) and (2) of that theorem. 
Proof of Theorem 1.3. For a suitable choice of the constant $L>0$ we have seen that

$$
\psi_{1}(z):=-\left(-\psi(z) e^{-L|z|^{2}}\right)^{1-1 / n}
$$

satisfies condition (1) of Theorem 1.1 and also condition (3) of Theorem 1.3, but with $M$ replaced by $M^{\prime}:=(1-1 / n) M$ and $N$ by $N^{\prime}:=(1-1 / n) N$. Let $z^{0} \in K$. From Lemma 3.3 we have, for any $0<r<\min \left\{r_{*}, \delta_{D}\left(z^{0}\right)\right\}$,

$$
\sup _{x \in B\left(z^{0}, r\right)} \mathscr{G}_{D}(x, w) \geq-\frac{\widetilde{C}_{n}}{\delta_{D}\left(z^{0}\right)^{2 n}}\left|\psi_{1}(w)\right|^{1 / n} \log \frac{C_{2}}{r^{n}} .
$$

First we use part (a) of Lemma 3.5, applied to $\psi_{1}$ : By condition (3) we find

$$
\frac{\log \left(2 R_{D} / \delta_{D}(w)\right)}{\inf _{B\left(w, \delta_{D}(w) / 2\right)}\left|\psi_{1}\right|} \leq C_{13}\left|\psi_{1}(w)\right|^{-\left(M^{\prime}+1\right) / N^{\prime}}
$$

and hence

$$
\mathscr{G}_{D}(z, w) \geq C_{13} \frac{\psi_{1}(z)}{\left|\psi_{1}(w)\right|^{\left(M^{\prime}+1\right) / N^{\prime}}},
$$

provided that $|z-w| \geq \delta_{D}(w) / 2$. We want to modify the proof of Lemma 4.3 in order to estimate the left-hand side from above for those $z^{0} \in D$ for which $\mathscr{G}_{D}\left(z^{0}, w\right) \leq-C_{13}\left|\psi_{1}(w)\right|^{\beta}$.

For this purpose we define $S:=\left\{\left.x \in D\left|\mathscr{G}_{D}(x, w)<-C_{13}\right| \psi_{1}(w)\right|^{\beta}\right\}$ and

$$
v(x):= \begin{cases}\mathscr{G}_{D}(x, w) & \text { for } x \in D \backslash S, \\ \max \left\{\mathscr{G}_{D}(x, w), \widetilde{v}_{w}(x)\right\} & \text { for } x \in S,\end{cases}
$$

where $r_{w}$ is the distance of $\partial S$ to the boundary of $D$, and $\widetilde{v}_{w}$ is defined by

$$
\widetilde{v}_{w}(x):=\left(\mathscr{G}_{D}(\cdot, w)\right)_{m}(x)-\frac{1}{m} \log \frac{C_{2}}{r_{w}^{n}}-C_{13}\left|\psi_{1}(w)\right|^{\beta},
$$

where $C_{2}$ is the constant from (2.1). Then $v$ is plurisubharmonic, and $v \leq$ $(1-n / m) \mathscr{G}_{D}(\cdot, w)$. We want to estimate $\log \left(1 / r_{w}\right)$ from above. For this purpose let $x \in \partial S$ with $\delta_{D}(x)<1$. If $|x-w| \leq \delta_{D}(w) / 2$ we have $\delta_{D}(x) \geq$ $\delta_{D}(w) / 2$, and hence $\left(\right.$ since $\left.\delta_{D}(w)<1 / 2\right)$

$$
\log \frac{1}{\delta_{D}(x)} \leq \log \frac{2}{\delta_{D}(w)} \leq 2 \log \frac{1}{\delta_{D}(w)} \leq 2\left(\frac{C_{2}}{|\psi(w)|}\right)^{1 / N} .
$$

Now assume that $|x-w| \geq \delta_{D}(w) / 2$. Then

$$
-C_{13}\left|\psi_{1}(w)\right|^{\beta}=\mathscr{G}_{D}(x, w) \geq-C_{13} \frac{\left|\psi_{1}(x)\right|}{\left|\psi_{1}(w)\right|^{\left(M^{\prime}+1\right) / N^{\prime}}} .
$$

This implies

$$
\log \frac{1}{\delta_{D}(x)} \leq C_{14}\left|\psi_{1}(w)\right|^{-\frac{n}{N(n-1)}\left(\beta+\frac{M^{\prime}+1}{N^{\prime}}\right)}
$$


Trivially, this holds if $x \in S$ and $\delta_{D}(x) \geq 1$. In particular, if $\delta_{D}(w) \leq \delta_{*} \ll 1$, then

$$
\log \frac{C_{2}}{r_{w}^{n}} \leq C_{15}\left|\psi_{1}(w)\right|^{-1 / n+2 \gamma},
$$

because

$$
-\frac{n}{N(n-1)}\left(\beta+\frac{M^{\prime}+1}{N^{\prime}}\right)=-\frac{1}{n}+2 \gamma .
$$

Let $K \subset D$ be a compact set and $z^{0} \in K$ and $w \in D \backslash K$ with $\delta_{D}(w) \leq$ $\min \left\{\delta_{*}, \delta_{D}(K) / 4\right\}$. For $z_{*} \in B\left(z^{0}, \delta_{D}\left(z^{0}\right) / 2\right)$ we can estimate $\mathscr{G}_{D}\left(z_{*}, w\right)$ in terms of $\mathscr{G}_{D}\left(z^{0}, w\right)$ and $|\psi(w)|$. Let us assume that $\mathscr{G}_{D}\left(z^{0}, w\right) \leq-C_{13}|\psi(w)|^{\beta}$. As in the proof of Lemma 4.3, we have

$$
\begin{aligned}
\mathscr{G}_{D}\left(z_{*}, w\right) \leq & \left(\mathscr{G}_{D}(\cdot, w)\right)_{m}\left(z_{*}\right)+\frac{1}{C_{1} m} \\
= & \left(\mathscr{G}_{D}(\cdot, w)\right)_{m}\left(z^{0}\right)+\frac{1}{C_{1} m}+\left(\mathscr{G}_{D}(\cdot, w)\right)_{m}\left(z_{*}\right)-\left(\mathscr{G}_{D}(\cdot, w)\right)_{m}\left(z^{0}\right) \\
\leq & v\left(z^{0}\right)+\frac{1}{m} \log \frac{C_{2}}{r_{w}^{n}}+C_{13}\left|\psi_{1}(w)\right|^{\beta}+\frac{1}{C_{1} m} \\
& +\left(\mathscr{G}_{D}(\cdot, w)\right)_{m}\left(z_{*}\right)-\left(\mathscr{G}_{D}(\cdot, w)\right)_{m}\left(z^{0}\right) \\
\leq & \left(1-\frac{n}{m}\right) \mathscr{G}_{D}\left(z^{0}, w\right)+\frac{1}{m} \log \frac{C_{2}}{r_{w}^{n}}+C_{13}\left|\psi_{1}(w)\right|^{\beta}+\frac{1}{C_{1} m} \\
& +\left(\mathscr{G}_{D}(\cdot, w)\right)_{m}\left(z_{*}\right)-\left(\mathscr{G}_{D}(\cdot, w)\right)_{m}\left(z^{0}\right) \\
\leq & \mathscr{G}_{D}\left(z^{0}, w\right)+\frac{n}{m} \log \frac{R_{D}}{\left|z_{*}-w\right|}+\frac{C_{15}}{m}\left|\psi_{1}(w)\right|^{-1 / n+2 \gamma}+C_{13}\left|\psi_{1}(w)\right|^{\beta} \\
& +\frac{1}{C_{1} m}+\left(\mathscr{G}_{D}(\cdot, w)\right)_{m}\left(z_{*}\right)-\left(\mathscr{G}_{D}(\cdot, w)\right)_{m}\left(z^{0}\right) .
\end{aligned}
$$

We note that

$$
\log \frac{R_{D}}{\left|z_{*}-w\right|} \leq \log \frac{R_{D}}{\left|z^{0}-w\right|-\left|z_{*}-z^{0}\right|} \leq \log \frac{8 R_{D}}{5 \delta_{D}\left(z^{0}\right)} .
$$

Next choose

$$
m:=\frac{1}{\log \left(2 n R_{D} / \delta_{D}\left(z^{0}\right)\right)}\left|\psi_{1}(w)\right|^{-1 / n+\gamma} .
$$

From Lemma 4.2 we get, as in the proof of Lemma 4.3,

$$
\begin{aligned}
\mid\left(\mathscr{G}_{D}(\cdot, w)\right)_{m}\left(z_{*}\right)- & \left(\mathscr{G}_{D}(\cdot, w)\right)_{m}\left(z^{0}\right) \mid \\
& \leq C_{0}^{\prime} \frac{1}{m}\left(\frac{2 n R_{D}}{\delta_{D}\left(z^{0}\right)}\right)^{m+1} \frac{\left|z_{*}-z^{0}\right|}{\delta_{D}\left(z^{0}\right)^{n+1}} \\
& \leq C_{16}\left|\psi_{1}(w)\right|^{1 / n-\gamma} \frac{\left|z_{*}-z^{0}\right|}{\delta_{D}\left(z^{0}\right)^{n+2}} \exp \left(\left|\psi_{1}(w)\right|^{-1 / n+\gamma}\right) .
\end{aligned}
$$


For $z_{*} \in B\left(z^{0}, \delta_{D}\left(z^{0}\right) / 8\right)$ (after possibly shrinking $\delta_{*}$ ) this yields

$$
\begin{aligned}
\mathscr{G}_{D}\left(z_{*}, w\right) \leq & \mathscr{G}_{D}\left(z^{0}, w\right)+C_{17} \frac{\left(1+r \exp \left(\left|\psi_{1}(w)\right|^{-1 / n+\gamma}\right)\right)}{\delta_{D}\left(z^{0}\right)^{n+2}}\left|\psi_{1}(w)\right|^{1 / n-\gamma} \\
& +C_{17}^{\prime}\left|\psi_{1}(w)\right|^{\beta_{1}}
\end{aligned}
$$

with $\beta_{1}=\min \{\beta, 1 / n-\gamma\}$. Now we choose the radius $r$ from (6.1) as $r=$ $\exp \left(-\left|\psi_{1}(w)\right|^{-1 / n+\gamma}\right)$. Inserting this into (6.1) we find

$$
\begin{aligned}
\mathscr{G}_{D}\left(z^{0}, w\right) & +C_{18} \frac{\left|\psi_{1}(w)\right|^{1 / n-\gamma}}{\delta_{D}\left(z^{0}\right)^{n+2}}+C_{17}^{\prime}\left|\psi_{1}(w)\right|^{\beta_{1}} \\
& \geq \sup _{x \in B\left(z^{0}, r\right)} \mathscr{G}_{D}(x, w) \geq-\frac{\widetilde{C}_{n}}{\delta_{D}\left(z^{0}\right)^{2 n}}\left|\psi_{1}(w)\right|^{1 / n} \log \frac{C_{2}}{r^{n}} \geq-\frac{C_{19}\left|\psi_{1}(w)\right|^{\gamma}}{\delta_{D}\left(z^{0}\right)^{2 n}}
\end{aligned}
$$

if $\delta_{D}(w) \leq \delta_{*} \ll 1$.

This proves (because of $\gamma<1 / 2 n$ )

$$
\left|\mathscr{G}_{D}\left(z^{0}, w\right)\right| \leq \frac{C_{20}\left|\psi_{1}(w)\right|^{\gamma}}{\delta_{D}\left(z^{0}\right)^{2 n}}+C_{17}^{\prime}\left|\psi_{1}(w)\right|^{\beta_{1}}
$$

whenever $z^{0} \in K$ and $\mathscr{G}_{D}\left(z^{0}, w\right) \leq-C_{13}\left|\psi_{1}(w)\right|^{\beta}$. (Again, we tacitly supposed that $\delta_{D}(K)<1$, which is allowed.) For those $z^{0}$ for which $\mathscr{G}_{D}\left(z^{0}, w\right)$ $\geq-C_{13}\left|\psi_{1}(w)\right|^{\beta}$ there is nothing to be done.

The proof of Theorem 1.3 is complete.

7. The case $n=1$. In the one-dimensional case things are much easier, since the pluricomplex Green function equals the classical one and, in particular, it is symmetric.

TheOREM 7.1. Let $D \subset \mathbb{C}$ be a bounded hyperconvex domain and $\varphi_{D}$ be as in the introduction. Then

$$
\mathscr{G}_{D}(z, w) \geq-\frac{1}{2} \log \left(1+4 \frac{\left|\varphi_{D}(w)\right|}{|z-w|^{2}}\right) .
$$

Proof. Let $z, w \in D$ be different points. Then the function

$$
\phi(x):=\varphi_{D}(x)-\frac{1}{4}|x-w|^{2}
$$

is subharmonic on $D$ and negative. Also (since $\varphi_{D}<0$ )

$$
\frac{|x-w|^{2}}{-4 \phi(x)}<1
$$

As the left-hand side is logarithmically subharmonic, the function

$$
\frac{1}{2} \log \frac{|x-w|^{2}}{-4 \phi(x)}=\log |x-w|-\frac{1}{2} \log (-4 \phi(x))
$$


is a candidate for $\mathscr{G}_{D}(\cdot, w)$, and for $x=z$ we obtain

$$
\mathscr{G}_{D}(z, w) \geq-\frac{1}{2} \log \frac{-4 \phi(z)}{|z-w|^{2}}=-\frac{1}{2} \log \left(1+4 \frac{\left|\varphi_{D}(z)\right|}{|z-w|^{2}}\right) .
$$

By symmetry,

$$
\mathscr{G}_{D}(z, w)=\mathscr{G}_{D}(w, z) \geq-\frac{1}{2} \log \left(1+4 \frac{\left|\varphi_{D}(w)\right|}{|z-w|^{2}}\right) .
$$

\section{References}

[1] H. Bauer, Wahrscheinlichkeitstheorie, de Gruyter, Berlin, 1968.

[2] E. Bedford and J.-P. Demailly, Two counterexamples concerning the pluricomplex Green function in $\mathbb{C}^{n}$, Indiana Univ. Math. J. 37 (1988), 865-867.

[3] B. Berndtsson, The extension theorem of Ohsawa-Takegoshi and the theorem of Donnelly-Fefferman, Ann. Inst. Fourier (Grenoble) 46 (1996), 1083-1094.

[4] Z. Błocki, The Bergman metric and the pluricomplex Green function, Trans. Amer. Math. Soc. 357 (2005), 2613-2625.

[5] —, Estimates for the complex Monge-Ampère operator, Bull. Polish Acad. Sci. Math. 41 (1993), 151-157.

[6] - , The complex Monge-Ampère operator in hyperconvex domains, Ann. Scuola Norm. Sup. Pisa Cl. Sci. 23 (1996), 721-747.

[7] Z. Błocki and P. Pflug, Hyperconvexity and Bergman completeness, Nagoya Math. J. 151 (1998), 221-225.

[8] M. Carlehed, U. Cegrell and F. Wikström, Jensen measures, hyperconvexity and boundary behaviour of the pluricomplex Green function, Ann. Polon. Math. 71 (1999), 87-103.

[9] J.-P. Demailly, Mesures de Monge-Ampère et mesures pluriharmoniques, Math. Z. 194 (1987), 519-564.

[10] —, Regularization of closed positive currents and intersection theory, J. Algebraic Geom. 1 (1992), 361-409.

[11] K. Diederich and J. E. Fornæss, Pseudoconvex domains: Bounded strictly plurisubharmonic exhaustion functions, Invent. Math. 39 (1977), 129-141.

[12] K. Diederich and G. Herbort, Quantitative estimates for the Green function and an application to the Bergman metric, Ann. Inst. Fourier (Grenoble) 50 (2000), 1205-1228.

[13] —, - An alternative proof of a theorem of T. Ohsawa, Michigan Math. J. 46 (1999), 347-360.

[14] K. Diederich and T. Ohsawa, An estimate for the Bergman distance on pseudoconvex domains, Ann. of Math. 141 (1995), 181-190.

[15] G. Herbort, The Bergman metric on hyperconvex domains, Math. Z. 232 (1999), 183-196.

[16] - The pluricomplex Green function on pseudoconvex domains with a smooth boundary, Int. J. Math. 11 (2000), 509-523.

[17] - Localization lemmas for the Bergman metric at plurisubharmonic peak points, Nagoya Math. J. 171 (2003), 107-125. 
[18] M. Klimek, Extremal plurisubharmonic functions and invariant pseudodistances, Bull. Soc. Math. France 113 (1985), 231-240.

[19] P. Lelong, Fonctions plurisousharmoniques et formes différentielles positives, Gordon and Breach, Paris, 1968.

[20] T. Ohsawa and K. Takegoshi, On the extension of $L^{2}$-holomorphic functions, Math. Z. 195 (1987), 197-204.

[21] J.-L. Stehlé, Fonctions plurisousharmoniques et convexité holomorphe de certains fibrés analytiques, C. R. Acad. Sci. Paris Sér. A 279 (1974), 235-238.

[22] N. Trudinger, On imbeddings into Orlicz spaces and some applications, J. Math. Mech. 17 (1967), 473-483.

Fachbereich C - Mathematik und Naturwissenschaften

Bergische Universität Wuppertal

Gaußstraße 20

D-42097 Wuppertal, Germany

E-mail: gregor@math.uni-wuppertal.de

Received 28.1.2008

and in final form 25.6.2008 